\title{
Lower Bounds for the Size of Random Maximal $H$-Free Graphs
}

\author{
Guy Wolfovitz \\ Department of Computer Science \\ Haifa University, Haifa, Israel \\ gwolfovi@cs.haifa.ac.il
}

Submitted: Jun 19, 2008; Accepted: Dec 15, 2008; Published: Jan 7, 2009

Mathematics Subject Classifications: 05C80, 05C35

\begin{abstract}
We consider the next random process for generating a maximal $H$-free graph: Given a fixed graph $H$ and an integer $n$, start by taking a uniformly random permutation of the edges of the complete $n$-vertex graph $K_{n}$. Then, traverse the edges of $K_{n}$ according to the order imposed by the permutation and add each traversed edge to an (initially empty) evolving $n$-vertex graph - unless its addition creates a copy of $H$. The result of this process is a maximal $H$-free graph $\mathbb{M}_{n}(H)$. Our main result is a new lower bound on the expected number of edges in $\mathbb{M}_{n}(H)$, for $H$ that is regular, strictly 2-balanced. As a corollary, we obtain new lower bounds for Turán numbers of complete, balanced bipartite graphs. Namely, for fixed $r \geq 5$, we show that $\operatorname{ex}\left(n, K_{r, r}\right)=\Omega\left(n^{2-2 /(r+1)}(\ln \ln n)^{1 /\left(r^{2}-1\right)}\right)$. This improves an old lower bound of Erdős and Spencer.

Our result relies on giving a non-trivial lower bound on the probability that a given edge is included in $\mathbb{M}_{n}(H)$, conditioned on the event that the edge is traversed relatively (but not trivially) early during the process.
\end{abstract}

\section{Introduction}

Consider the next random process for generating a maximal $H$-free graph. Given $n \in \mathbb{N}$ and a graph $H$, assign every edge $f$ of the complete $n$-vertex graph $K_{n}$ a birthtime $\beta(f)$, distributed uniformly at random in the interval $[0,1]$. (Note that with probability 1 the birthtimes are distinct and so $\beta$ is a permutation.) Now start with the empty $n$-vertex graph and iteratively add edges to it as follows. Traverse the edges of $K_{n}$ in order of their birthtimes, starting with the edge whose birthtime is smallest, and add each traversed edge to the evolving graph, unless its addition creates a copy of $H$. When all edges of $K_{n}$ have been exhausted, the process ends. Denote by $\mathbb{M}_{n}(H)$ the graph which is the result of the above process. The main concern in this paper is bounding from below the 
expected number of edges of $\mathbb{M}_{n}(H)$, which is denoted by $e\left(\mathbb{M}_{n}(H)\right)$. We always think of $H$ as being fixed and of $n$ as going to $\infty$. To be able to state our results, we need a few definitions. For a graph $H$, let $v_{H}$ and $e_{H}$ denote, respectively, the number of vertices and edges in $H$. Say that a graph $H$ is strictly 2-balanced if $v_{H}, e_{H} \geq 3$ and for every $F \subsetneq H$ with $v_{F} \geq 3,\left(e_{H}-1\right) /\left(v_{H}-2\right)>\left(e_{F}-1\right) /\left(v_{F}-2\right)$. Examples of strictly 2-balanced graphs include the $r$-cycle $C_{r}$, the complete $r$-vertex graph $K_{r}$, the complete bipartite graph $K_{r-1, r-1}$ and the $(r-1)$-dimensional cube, for all $r \geq 3$. Note that all of these examples are of graphs which are regular. Our main result follows.

Theorem 1.1. Let $H$ be a regular, strictly 2-balanced graph. Then

$$
\mathbb{E}\left[e\left(\mathbb{M}_{n}(H)\right)\right]=\Omega\left(n^{2-\left(v_{H}-2\right) /\left(e_{H}-1\right)}(\ln \ln n)^{1 /\left(e_{H}-1\right)}\right) .
$$

Before discussing what was previously known about $e\left(\mathbb{M}_{n}(H)\right)$, we state an immediate consequence of Theorem 1.1 in extremal graph theory. Let $\operatorname{ex}(n, H)$ be the largest integer $m$ such that there exists an $H$-free graph over $n$ vertices and $m$ edges. For the case where $H=K_{r, r}$, Kövári, Sós and Turán proved that for fixed $r, \operatorname{ex}\left(n, K_{r, r}\right)=O\left(n^{2-1 / r}\right)$. For $r \in\{2,3\}$ this upper bound is known to be tight, by explicit constructions, due to Erdős, Rényi and Sós [4] and Brown [3]. Since $\operatorname{ex}\left(n, K_{4,4}\right) \geq \operatorname{ex}\left(n, K_{3,3}\right)$, one has that $\operatorname{ex}\left(n, K_{4,4}\right)=\Omega\left(n^{2-1 / 3}\right)$. For fixed $r \geq 5$, Erdős and Spencer [5] used a simple application of the probabilistic method to prove $\operatorname{ex}\left(n, K_{r, r}\right)=\Omega\left(n^{2-2 /(r+1)}\right)$. Now note that Theorem 1.1 implies a lower bound for ex $(n, H)$ for every regular, strictly 2-balanced graph. Hence, since $K_{r, r}$ is regular and strictly 2-balanced, we obtain the next lower bound on $\operatorname{ex}\left(n, K_{r, r}\right)$ which improves asymptotically the lower bound of Erdös and Spencer for $r \geq 5$.

Theorem 1.2. For all $r \geq 5$, ex $\left(n, K_{r, r}\right)=\Omega\left(n^{2-2 /(r+1)}(\ln \ln n)^{1 /\left(r^{2}-1\right)}\right)$.

\subsection{Previous bounds on $e\left(\mathbb{M}_{n}(H)\right)$}

The first to investigate the number of edges in $\mathbb{M}_{n}(H)$ were Ruciński and Wormald [10], who considered the case where $H=K_{1, r+1}$ is a star with $r+1$ edges. In that case, it was shown than with probability approaching 1 as $n$ goes to infinity, $\mathbb{M}_{n}(H)$ is an extremal $H$-free graph (that is, every vertex in $\mathbb{M}_{n}(H)$ has degree exactly $r$, except perhaps for at most one vertex whose degree is $r-1)$. Erdős, Suen and Winkler [6] showed that with probability that goes to 1 as $n$ goes to $\infty, e\left(\mathbb{M}_{n}\left(K_{3}\right)\right)=\Omega\left(n^{3 / 2}\right)$. Bollobás and Riordan [2] considered the case of $H \in\left\{K_{4}, C_{4}\right\}$, and showed that with probability that goes to 1 as $n$ goes to $\infty, e\left(\mathbb{M}_{n}\left(K_{4}\right)\right)=\Omega\left(n^{8 / 5}\right)$ and $e\left(\mathbb{M}_{n}\left(C_{4}\right)\right)=\Omega\left(n^{4 / 3}\right)$. Osthus and Taraz [9] generalized these bounds for every strictly 2-balanced graph $H$, showing that with probability that goes to 1 as $n$ goes to $\infty, e\left(\mathbb{M}_{n}(H)\right)=\Omega\left(n^{2-\left(v_{H}-2\right) /\left(e_{H}-1\right)}\right)$. Note that the above lower bounds trivially imply similar lower bounds on the expectation of $e\left(\mathbb{M}_{n}(H)\right)$. It is worth mentioning that all of the above lower bounds on the expectation of $e\left(\mathbb{M}_{n}(H)\right)$ can be derived using standard correlation inequalities.

The first non-trivial lower bound on the expectation of $e\left(\mathbb{M}_{n}(H)\right)$ for some graph $H$ that contains a cycle was given by Spencer [12]. Spencer showed that for every constant 
$a$ there exists $n_{0}=n_{0}(a)$ such that for every $n \geq n_{0}, \mathbb{E}\left[e\left(\mathbb{M}_{n}\left(K_{3}\right)\right)\right] \geq a n^{3 / 2}$. In the same paper, Spencer conjectured that $\mathbb{E}\left[e\left(\mathbb{M}_{n}\left(K_{3}\right)\right)\right]=\Theta\left(n^{3 / 2}(\ln n)^{1 / 2}\right)$. Recently, Bohman [1] resolved Spencer's conjecture, showing that indeed $\mathbb{E}\left[e\left(\mathbb{M}_{n}\left(K_{3}\right)\right)\right]=\Theta\left(n^{3 / 2}(\ln n)^{1 / 2}\right)$. Bohman also proved a lower bound of $\Omega\left(n^{8 / 5}(\ln n)^{1 / 5}\right)$ for the expected number of edges in $\mathbb{M}_{n}\left(K_{4}\right)$. In fact, Bohman's lower bounds hold with probability that goes to 1 as $n$ goes to $\infty$. We discuss Bohman's argument and compare it to ours below.

As for upper bounds: The currently best upper bound on the expectation of $e\left(M_{n}(H)\right)$, for $H$ that is strictly 2-balanced over at least 4 vertices is, by a result of Osthus and Taraz [9], at most $O\left(n^{2-\left(v_{H}-2\right) /\left(e_{H}-1\right)}(\ln n)^{1 /\left(\Delta_{H}-1\right)}\right)$, where $\Delta_{H}$ denotes the maximum degree of $H$.

\subsection{Overview of the proof of Theorem 1.1}

Let $H$ be a regular, strictly 2-balanced graph. We would like to analyse the random process generating $\mathbb{M}_{n}(H)$. In order to do this-and the reason will soon be apparent-it would be convenient for us to think slightly differently about the definition of $\beta$. Let $\mathbb{G}(n, \rho)$ be the standard Erdös-Rényi random graph, which is defined by keeping every edge of $K_{n}$ with probability $\rho$, independently of the other edges. Then an alternative, equivalent definition of $\beta$ is this: For every edge $f \in \mathbb{G}(n, \rho)$ assign uniformly at random a birthtime $\beta(f) \in[0, \rho]$, and for every edge $f \in K_{n} \backslash \mathbb{G}(n, \rho)$ assign uniformly at random a birthtime $\beta(f) \in(\rho, 1]$. Clearly, in this definition, every edge $f \in K_{n}$ is assigned a uniformly random birthtime $\beta(f) \in[0,1]$ and so this new definition is equivalent to the original definition of $\beta$. Note that $\mathbb{G}(n, \rho)$ denotes here the set of edges in $K_{n}$ whose birthtime is at most $\rho$. The main advantage of this new view of $\beta$ is that in order to analyse the event $\left\{f \in \mathbb{M}_{n}(H) \mid \beta(f)<\rho^{\prime}\right\}$ for some $\rho^{\prime} \leq \rho$, it is enough to consider only the distribution of the birthtimes of edges of $\mathbb{G}(n, \rho)$. Hopefully, for our choice of $\rho, \mathbb{G}(n, \rho)$ will be structured enough so that we could take advantage of the structures appearing in it and use them to find a non-trivial lower bound on the probability of $\left\{f \in \mathbb{M}_{n}(H) \mid \beta(f)<\rho^{\prime}\right\}$. This is the basic idea of the proof. We next describe, informally, what structures in $\mathbb{G}(n, \rho)$ we hope to take advantage of in order to prove Theorem 1.1.

For an edge $f \in K_{n}$, let $\Lambda(f, \rho)$ be the set of all $G \subseteq \mathbb{G}(n, \rho) \backslash\{f\}$ such that $G \cup\{f\}$ is isomorphic to $H$. Fix an edge $f \in K_{n}$ and let $\rho^{\prime} \leq \rho$. Assume that the event $\left\{\beta(f)<\rho^{\prime}\right\}$ occurs. Suppose now that we want to estimate the probability of the event $\left\{f \in \mathbb{M}_{n}(H)\right\}$, which, by linearity of expectation, is essentially what we need to do in order to prove Theorem 1.1. We seek a sufficient condition for the event $\left\{f \in \mathbb{M}_{n}(H)\right\}$. One such trivial event is this: Say that $f$ survives-trivially if for every graph $G \in \Lambda(f, \rho)$ there exists an edge $g \in G$ such that $\{\beta(g)>\beta(f)\}$ occurs. Clearly if $f$ survives-trivially then we have $\left\{f \in \mathbb{M}_{n}(H)\right\}$. We can improve this simple sufficient condition as follows. Say that an edge $g$ doesn't survive if there exists $G^{\prime} \in \Lambda(g, \rho)$ such that for every edge $g^{\prime} \in G^{\prime}$ we have $\left\{\beta\left(g^{\prime}\right)<\beta(g)\right\}$ and $g^{\prime}$ survives-trivially. Note that if $g$ doesn't survive then $\left\{g \notin \mathbb{M}_{n}(H)\right\}$ occurs. Now say that $f$ survives if for every graph $G \in \Lambda(f, \rho)$ there exists an edge $g \in G$ such that either $\{\beta(g)>\beta(f)\}$ or $g$ doesn't survive. Then the event that $f$ survives implies $\left\{f \in \mathbb{M}_{n}(H)\right\}$. 
Observe that the event that $f$ survives was defined above using an underlying tree-like structure of constant depth, in which the root is $f$, the set of children of any non-leaf edge $g$ is $\Lambda(g, \rho)$ and for any $G \in \Lambda(g, \rho)$, the set of children of $G$ is simply the set of edges in $G$. Using the same idea as in the previous paragraph, we could have defined the event that $f$ survives using an underlying tree-like structure which is much deeper than the constant depth tree-like structure that was used above. Intuitively, the deeper this tree-like structure is - the better the chances are for $f$ to survive. Therefore, we would be interested in defining the event that $f$ survives using a rather deep underlying tree-like structure. We will then be interested in lower bounding the probability that $f$ survives.

Now, in order to analyse the event that $f$ survives, it would be useful if the underlying tree-like structure $T$ is good in the following sence: Every edge that appears in $T$ appears exactly once ${ }^{\dagger}$. The advantage of $T$ being good is that for many of the edges that appear in $T$, the events that these edges survive or doesn't survive are pairwise independent. This property can be used to analyse recursively the event that $f$ survives. Hence, it would be very helpful if we can show that $T$ is good with high probability. Showing this is a key ingredient of our proof.

Given the informal discussion above, the proof of Theorem 1.1 looks very roughly as follows. At the first part of the proof we consider the graph $\mathbb{G}(n, \rho)$ for a relatively large $\rho$, and show that for a fixed edge $f \in K_{n}$, with probability that approaches 1 as $n$ goes to $\infty$, we can associate with $f$ a tree $T$ which is similar to the tree-like structure described above and which is both good and deep. Then, the second part has this structure: We assume first that $\left\{\beta(f)<\rho^{\prime}\right\}$ occurs for some suitably chosen $\rho^{\prime} \leq \rho$. We also assume that the tree $T$ that is associated with $f$ is good and deep, which occurs with high probability. Then, we associate with $f$ and $T$ an event which is essentially the event that $f$ survives, as described informally above, and argue that this event implies $\left\{f \in \mathbb{M}_{n}(H)\right\}$. Lastly, we give an explicit lower bound on the probability of the event that we have associated with $f$ and $T$. This will give us a lower bound on the probability of $\left\{f \in \mathbb{M}_{n}(H)\right\}$ conditioned on $\left\{\beta(f)<\rho^{\prime}\right\}$. For our choice of $\rho^{\prime}$, this will imply Theorem 1.1.

\subsubsection{Comparison with previous work}

The basic idea that we have outlined in the overview above was used already by Erdös, Suen and Winkler [6] and by Spencer [12] for the case $H=K_{3}$. (Their results have been mentioned above.) In [6], the authors have analyzed the event that an edge $f$ survivestrivially, as described above, and considered implicitly the graph $\mathbb{G}(n, 1)$. This elementary argument gives a reasonable lower bound on the probability of $\left\{f \in \mathbb{M}_{n}\left(K_{3}\right) \mid \beta(f)<\right.$ $\left.a n^{-1 / 2}\right\}$, for small constant $a$ (e.g., $\left.a=1\right)$. In $[12]$ the graph $\mathbb{G}(n, 1)$ was again considered implicitly, but a more general event - essentially the event that an edge $f$ survives, with an underlying tree-like structure of constant depth - was analyzed; Using this, Spencer

\footnotetext{
${ }^{\dagger}$ In this informal discussion, we cannot hope that $T$ would be good, since for example, $f$ appears as an edge in some $G^{\prime} \in \Lambda(g, \rho)$ for some $g \in G \in \Lambda(f, \rho)$. We will define in Section 2 the tree $T$ slightly differently, so that this situation is avoided, while still maintaining that if $f$ survives then $\left\{f \in \mathbb{M}_{n}(H)\right\}$ occurs. Yet, for the purpose of communicating the idea of the proof, it would be useful to assume that $T$ could be good.
} 
was able to give a lower bound on the probability of $\left\{f \in \mathbb{M}_{n}\left(K_{3}\right) \mid \beta(f)<a n^{-1 / 2}\right\}$, for $a$ being arbitrary large, but constant independent of $n$. As we have discussed above, we consider explicitly the graph $\mathbb{G}(n, \rho)$ and we do that for some suitably chosen $\rho<1$. This is the key to our improvement. For example, for the case of $H=K_{3}$, this enables us to give a non-trivial lower bound on the probability of $\left\{f \in \mathbb{M}_{n}\left(K_{3}\right) \mid \beta(f)<a n^{-1 / 2}\right\}$, for $a=\left\lfloor(\ln n)^{1 / 24}\right\rfloor$. Moreover, our arguments apply for every other regular, strictly 2-balanced graph.

\subsubsection{Comparison with Bohman's argument}

As stated above, Bohman [1] have proved stronger bounds than those given in Theorem 1.1, for the case where $H \in\left\{K_{3}, K_{4}\right\}$. To do this, Bohman uses the differential equation method. The basic argument, applied for the case $H=K_{3}$, can be described as follows. First, a collection of random variables that evolve throughout the random process is introduced and tracked throughout the evolution of $\mathbb{M}_{n}\left(K_{3}\right)$. This collection includes, for example, the random variable $O_{i}$, which denotes the set of edges that have not yet been traversed by the process, and which can be added to the current graph without forming a triangle, after exactly $i$ edges have been added to the evolving graph. Now, at certain times during the process (i.e., at those times in which new edges are added to the evolving graph), Bohman expresses the expected change in the values of the random variables in the collection, using the same set of random variables. This allows one to express the random variables in the collection using the solution to an autonomous system of ordinary differential equations. The main technical effort in Bohman's work then shows that the random variables in the collection are tightly concentrated around the trajectory given by the solution to this system. The particular solution to the system then implies that with high probability $O_{I}$ is still large for $I:=n^{3 / 2}(\ln n)^{1 / 2} / 32$. This gives Bohman's lower bound on the expected number of edges in $\mathbb{M}_{n}\left(K_{3}\right)$.

We remark that Bohman's argument probably can be used to analyse the random process generating $\mathbb{M}_{n}(H)$ for $H \notin\left\{K_{3}, K_{4}\right\}$, and this can most likely lead to stronger lower bounds than those given in Theorem 1.1. In comparison with Bohman's argument, our argument is more direct in the sence that it considers a single edge and estimates directly the probability of it being included in $\mathbb{M}_{n}(H)$. We remark that our argument can be strengthened and generalized in the following way for the case $H=K_{3}$. One can use our basic argument so as to give an asymptotically tight expression for the probability that a fixed triangle-free graph $F$ is included in $\mathbb{M}_{n}\left(K_{3}\right)$, conditioned on the event that the edges of $F$ all have birthtimes which are relatively, but not trivially small. This, in turn, can be used to tackle the following question, which is left open even after Bohman's breakthrough. Suppose we trim the random process generating $\mathbb{M}_{n}\left(K_{3}\right)$ right after every edge whose birthtime is less than $c n^{-1 / 2}$ has been traversed, where $c=(\ln n)^{1 / 24}$. That is, let us consider the trimmed graph $\mathbb{M}_{n}\left(K_{3}\right) \cap\left\{f: \beta(f)<c n^{-1 / 2}\right\}$. We may ask what is the number of paths of length 2 in the trimmed graph. Bohman's argument does not answer this question, but rather places an upper bound of $\left(\begin{array}{l}n \\ 2\end{array}\right) \cdot(\ln n)^{2}$ on that number. Yet, the above-mentioned strengthening and generalization together with the second moment 
method can be used to show that the number of paths of length 2 in the trimmed graph is concentrated around $\left(\begin{array}{l}n \\ 2\end{array}\right) \cdot \ln c$. Similarly, one can prove concentration results for the number of small cycles in the trimmed graph.

\subsection{Organization of the paper}

In Section 2 we give the basic definitions we use throughout the paper and in particular, we give the formal definition of what we have referred to above as a good tree-like structure. We also state in Section 2 the two main lemmas we prove throughout the paper and argue that these lemmas imply the validity of Theorem 1.1. The two main lemmas are proved in Sections 3 and 4 and these two sections correspond to the two parts of the proof that were sketched at Section 1.2.

\subsection{Basic notation and conventions}

We use $K_{n}$ to denote the complete graph over the vertex set $[n]:=\{1,2, \ldots, n\}$. We set $[0]:=\emptyset$. We use $f, g, g^{\prime}$ to denote edges of $K_{n}$ and $F, G, G^{\prime}$ to denote subgraphs of $K_{n}$ or subgraphs of any other fixed graph. Throughout the paper, the hidden constants in the big-O and big-Omega notation, are either absolute constants or depend only on an underlying fixed graph $H$ which should be understood from the context. If $x=x(n)$ and $y=y(n)$ are functions of $n$, we write $y=o(x)$ if $y / x$ goes to 0 as $n$ goes to $\infty$ and $y=\omega(x)$ if $y / x$ goes to $\infty$ as $n$ goes to $\infty$.

\section{Main lemmas and proof of Theorem 1.1}

In this section we give the overall structure of the proof of Theorem 1.1, including the required basic definitions and two key lemmas-whose validity imply the theorem. We fix once and for the rest of this paper a regular, strictly 2-balanced graph $H$ and prove Theorem 1.1 for that specific $H$. We always think of $n$ as being sufficiently large, and define the following functions of $n$.

Definition 1. Define

$$
\begin{aligned}
& k=k(n) \quad:=n^{(\ln n)^{-1 / 2}}, \\
& \rho=\rho(n):=k n^{-\left(v_{H}-2\right) /\left(e_{H}-1\right)}, \\
& c=c(n) \quad:=\left\lfloor(\ln n)^{1 /\left(8 e_{H}\right)}\right\rfloor, \quad \text { and } \\
& D=D(n) \quad:=2\left\lfloor(\ln n)^{1 / 4}\right\rfloor+1 \text {. }
\end{aligned}
$$

In order to prove Theorem 1.1, we will show that for our fixed graph $H$, and for every edge $f \in K_{n}$,

$$
\operatorname{Pr}\left[f \in \mathbb{M}_{n}(H) \mid \beta(f)<c n^{-\left(v_{H}-2\right) /\left(e_{H}-1\right)}\right]=\Omega\left(\frac{(\ln c)^{1 /\left(e_{H}-1\right)}}{c}\right) .
$$


Note that (1) implies Theorem 1.1: Since $\operatorname{Pr}\left[\beta(f)<c n^{-\left(v_{H}-2\right) /\left(e_{H}-1\right)}\right]=c n^{-\left(v_{H}-2\right) /\left(e_{H}-1\right)}$, it follows from (1) that for all $f \in K_{n}, \operatorname{Pr}\left[f \in \mathbb{M}_{n}(H)\right]=\Omega\left(n^{-\left(v_{H}-2\right) /\left(e_{H}-1\right)}(\ln c)^{1 /\left(e_{H}-1\right)}\right)$. Using the fact that $\ln c=\Omega(\ln \ln n)$ and using linearity of expectation, this last bound implies Theorem 1.1. It thus remains to prove (1). The rest of this section is devoted to outlining the proof of (1).

Recall that for an edge $f \in K_{n}$, we define $\Lambda(f, \rho)$ to be the set of all $G \subseteq \mathbb{G}(n, \rho) \backslash\{f\}$ such that $G \cup\{f\}$ is isomorphic to $H$. We now set up to define what we have referred to in the introduction as a good tree-like structure.

A rooted tree $T$ is a directed tree with a distinguished node, called the root, which is connected by a directed path to any other node in $T$. If $u$ is a node in $T$ then the set of nodes that are adjacent ${ }^{\ddagger}$ to $u$ in $T$ is denoted by $\Gamma_{T}(u)$. The height of a node $u$ in a rooted tree $T$ is the length of the longest path from $u$ to a leaf. The height of a rooted tree is the height of its root. We shall consider labeled (rooted) trees. If $u$ is a node in a labeled tree $T$, we denote by $L_{T}(u)$ the label of the node $u$ in $T$.

Definition $2\left(T_{f, d}\right)$. Let $f \in K_{n}$ and $d \in \mathbb{N}$. We define inductively a labeled, rooted tree $T_{f, d}$ of height $2 d$. The nodes at even distance from the root will be labeled with edges of $K_{n}$. The nodes at odd distance from the root will be labeled with subgraphs of $K_{n}$.

- $T_{f, 1}$ :

- The root $v_{0}$ of $T_{f, 1}$ is labeled with the edge $f$.

- For every subgraph $G_{1} \in \Lambda(f, \rho)$ : Set a new node $u_{1}$ which is adjacent to $v_{0}$ and whose label is $G_{1}$; Furthermore, for each edge $g \in G_{1}$ set a new node $v_{1}$ which is adjacent to $u_{1}$ and whose label is $g$.

- $T_{f, d}, d \geq 2$ : We construct the tree $T_{f, d}$ by adding new nodes to $T=T_{f, d-1}$ as follows. Let $\left(v_{0}, u_{1}, v_{1}, \ldots, u_{d-1}, v_{d-1}\right)$ be a directed path in $T_{f, d-1}$ from the root $v_{0}$ to a leaf $v_{d-1}$. Let $g_{d-1}=L_{T}\left(v_{d-1}\right)$ and $g_{d-2}=L_{T}\left(v_{d-2}\right)$. For every subgraph $G_{d} \in \Lambda\left(g_{d-1}, \rho\right)$ such that $g_{d-2} \notin G_{d}$ do: Set a new node $u_{d}$ which is adjacent to $v_{d-1}$ and whose label is $G_{d}$; Furthermore, for each edge $g_{d} \in G_{d}$ set a new node $v_{d}$ which is adjacent to $u_{d}$ and whose label is $g_{d}$.

Definition 3 (good tree). Let $f \in K_{n}$ and $d \in \mathbb{N}$. Consider the tree $T=T_{f, d}$ and let $v_{0}$ denote the root of $T$. We say that $T$ is good if the following three properties hold:

P1 If $G$ is the label of a node $u$ at odd distance from $v_{0}$ then $G \cap\{f\}=\emptyset$.

P2 If $G, G^{\prime}$ are the labels of two distinct nodes at odd distance from $v_{0}$ then $G \cap G^{\prime}=\emptyset$.

P3 If $g$ is the label of a non-leaf node $v$ at even distance from $v_{0}$ then $\left|\Gamma_{T}(v)\right|=$ $|\Lambda(g, \rho)|-O(1)$.

\footnotetext{
${ }^{\ddagger}$ We say that node $v$ is adjacent to node $u$ in a given directed graph, if there is a directed edge from $u$ to $v$.
} 
Recall the definition of $\rho$ and note that the expected size of $\Lambda(g, \rho)$ is $\lambda k^{e_{H}-1}$, where $\lambda=\lambda^{\prime}(1-o(1))$ and $\lambda^{\prime} \leq 1$ depends only on $H$. (This follows from the fact that for every edge $g \in K_{n}$, the cardinality of $\Lambda(g, 1)$ is between $\left(\begin{array}{c}n-2 \\ v_{H}-2\end{array}\right)$ and $\left(v_{H}-2\right) !\left(\begin{array}{c}n-2 \\ v_{H}-2\end{array}\right)$, and from the fact that for every $G \in \Lambda(g, 1)$, the probability of $\{G \in \Lambda(g, \rho)\}$ is $\rho^{e_{H}-1}$.) Define the event $E_{1}$ to be the event that for every edge $g \in K_{n}$,

$$
\lambda k^{e_{H}-1}-k^{e_{H} / 2-1 / 3} / 2 \leq|\Lambda(g, \rho)| \leq \lambda k^{e_{H}-1}+k^{e_{H} / 2-1 / 3} / 2 .
$$

For an edge $f \in K_{n}$, let $E_{2}(f)$ be the event that $T_{f, D}$ is good. The next lemma is proved in Section 3.

Lemma 2.1. For every edge $f \in K_{n}$,

$$
\operatorname{Pr}\left[E_{2}(f) \cap E_{1}\right]=1-o(1) .
$$

Assuming that the event $E_{2}(f) \cap E_{1}$ occurs, the tree $T_{f, D}$ is exactly what we have referred to informally in the introduction as a good tree-like structure. Assuming that such a structure exists in $\mathbb{G}(n, \rho)$, we derive in Section 4 a lower bound on the probability of $\left\{f \in \mathbb{M}_{n}(H)\right\}$, conditioned on $\left\{\beta(f)<c n^{-\left(v_{H}-2\right) /\left(e_{H}-1\right)}\right\}$. Formally, we prove the next lemma.

Lemma 2.2. For every edge $f \in K_{n}$,

$$
\operatorname{Pr}\left[f \in \mathbb{M}_{n}(H) \mid E_{2}(f) \cap E_{1}, \beta(f)<c n^{-\left(v_{H}-2\right) /\left(e_{H}-1\right)}\right]=\Omega\left(\frac{(\ln c)^{1 /\left(e_{H}-1\right)}}{c}\right) .
$$

Trivially, Lemmas 2.1 and 2.2 imply (1) and hence Theorem 1.1. Therefore, in order to prove Theorem 1.1, it remains to prove these two lemmas.

\section{Proof of Lemma 2.1}

The proof is divided to two parts. In the first part, given at Section 3.1, we lower bound the probability of the event $E_{1}$. In the second part we lower bound the probability of the event $E_{2}(f)$. Since these two lower bounds would be shown to be $1-o(1)$, Lemma 2.1 will follow.

\subsection{Bounding $\operatorname{Pr}\left[E_{1}\right]$}

In this subsection we show that the probability of the event $E_{1}$ is $1-o(1)$. In order to do this, since there are at most $n^{2}$ edges in $K_{n}$, it suffices to fix an edge $g \in K_{n}$ and show that the following two equalities hold:

$$
\begin{aligned}
& \operatorname{Pr}\left[|\Lambda(g, \rho)| \geq \lambda k^{e_{H}-1}-k^{e_{H} / 2-1 / 3} / 2\right]=1-n^{-\omega(1)}, \\
& \operatorname{Pr}\left[|\Lambda(g, \rho)| \leq \lambda k^{e_{H}-1}+k^{e_{H} / 2-1 / 3} / 2\right]=1-n^{-\omega(1)} .
\end{aligned}
$$

Throughout this section we will use several times the following fact. 
Fact 3.1. There exists a constant $\varepsilon_{H}>0$, that depends only on $H$, such that the following holds for all sufficiently large $n$ : If $F \subsetneq H$ and $v_{F} \geq 3$ then

$$
n^{v_{H}-v_{F}} \rho^{e_{H}-e_{F}} \leq n^{-\varepsilon_{H}} .
$$

Proof. Fix $F \subsetneq H$ with $v_{F} \geq 3$. Since $H$ is strictly 2-balanced, we have that $\left(e_{F}-1\right)\left(v_{H}-\right.$ $2) /\left(e_{H}-1\right)<v_{F}-2$. Hence, there exists a constant $\varepsilon_{H}^{\prime}>0$ such that $n^{-v_{F}+2} \rho^{-e_{F}+1}=$ $n^{-v_{F}+2+\left(e_{F}-1\right)\left(v_{H}-2\right) /\left(e_{H}-1\right)+o(1)} \leq n^{-\varepsilon_{H}^{\prime}+o(1)}$ (here we have also used the fact that $k=$ $\left.n^{o(1)}\right)$. We also note that $n^{v_{H}-2} \rho^{e_{H}-1}=k^{e_{H}-1}=n^{o(1)}$. Therefore, $n^{v_{H}-v_{F}} \rho^{e_{H}-e_{F}}=$ $n^{v_{H}-2-v_{F}+2} \rho^{e_{H}-1-e_{F}+1} \leq n^{-\varepsilon_{H}^{\prime}+o(1)}$. To complete the proof, take the subgraph $F \subsetneq H$ with $v_{F} \geq 3$ which minimizes $\varepsilon_{H}^{\prime}$ above, and for that particular $\varepsilon_{H}^{\prime}$, take $\varepsilon_{H}=\varepsilon_{H}^{\prime} / 2$.

We prove (2) and (3) in Sections 3.1.1 and 3.1.2, respectively.

\subsubsection{The lower tail}

For $G \in \Lambda(g, 1)$, let $X_{G}$ be the indicator random variable for the event $\{G \subseteq \mathbb{G}(n, \rho)\}$. Let $X=\sum_{G \in \Lambda(g, 1)} X_{G}$. Then $|\Lambda(g, \rho)|=X$ and $\mathbb{E}[X]=\lambda k^{e_{H}-1}$. Let $\Delta=\sum_{G, G^{\prime}} \mathbb{E}\left[X_{G} \cap X_{G^{\prime}}\right]$ where the sum ranges over all ordered pairs $G, G^{\prime} \in \Lambda(g, 1)$ with $G \cap G^{\prime} \neq \emptyset$ (this includes the pairs $G, G^{\prime}$ with $\left.G=G^{\prime}\right)$. Then from Janson [8] we have that for every $0 \leq t \leq \mathbb{E}[X]$,

$$
\operatorname{Pr}[X \leq \mathbb{E}[X]-t] \leq \exp \left(-\frac{t^{2}}{2 \Delta}\right) .
$$

We now bound $\Delta$ from above. In order to do this, first note that for every $F \subseteq H$ and for every $G \in \Lambda(g, 1)$, the number of subgraphs $G^{\prime} \in \Lambda(g, 1)$ such that $(G \cup\{g\}) \cap\left(G^{\prime} \cup\{g\}\right)$ is isomorphic to $F$ is at most $O\left(n^{v_{H}-v_{F}}\right)$. Also, the number of subgraphs $G \in \Lambda(g, 1)$ is trivially at most $n^{v_{H}-2}$. Hence, denoting by $\sum_{F}$ the sum over all $F \subseteq H$ with $v_{F} \geq 3$, the number of pairs $G, G^{\prime}$ which contribute to $\Delta$ is at most $\sum_{F} O\left(n^{2 v_{H}-v_{F}-2}\right)$. For every pair $G, G^{\prime}$ as above, if $(G \cup\{g\}) \cap\left(G^{\prime} \cup\{g\}\right)$ is isomorphic to $F$ then $\mathbb{E}\left[X_{G} \cap X_{G^{\prime}}\right]=\rho^{2 e_{H}-e_{F}-1}$. Hence

$$
\begin{aligned}
\Delta \leq \sum_{F} O\left(n^{2 v_{H}-v_{F}-2} \rho^{2 e_{H}-e_{F}-1}\right) & =\sum_{F} O\left(n^{2 v_{H}-4-v_{F}+2} \rho^{2 e_{H}-2-e_{F}+1}\right) \\
& =k^{2\left(e_{H}-1\right)} \sum_{F} O\left(n^{-v_{F}+2} \rho^{-e_{F}+1}\right) .
\end{aligned}
$$

Now if $F \subsetneq H$ and $v_{F} \geq 3$ then by the fact that $H$ is strictly 2-balanced we have $n^{-v_{F}+2} \rho^{-e_{F}+1} \leq n^{-\varepsilon_{H}^{\prime}+o(1)}$ for some $\varepsilon_{H}^{\prime}>0$ that depends only on $H$ (see the proof of Fact 3.1). If $F$ on the other hand satisfies $F=H$, then $n^{-v_{F}+2} \rho^{-e_{F}+1}=k^{-\left(e_{H}-1\right)}$. Hence, we can further upper bound (5) by $O\left(k^{e_{H}-1}\right)$. This upper bound on $\Delta$ can be used with (4) to show that

$$
\operatorname{Pr}\left[X \geq \mathbb{E}[X]-k^{e_{H} / 2-1 / 3} / 2\right] \geq 1-\exp \left(-\Omega\left(k^{1 / 3}\right)\right) .
$$

This gives us (2). 


\subsubsection{The upper tail}

We are interested in giving a lower bound on the probability of the event that $|\Lambda(g, \rho)| \leq$ $\lambda k^{e_{H}-1}+k^{e_{H} / 2-1 / 3} / 2$. The technique we use is due to Spencer [11]. Let $\mathcal{G}$ be the graph over the vertex set $\Lambda(g, \rho)$ and whose edge set consists of all pairs of distinct vertices $G, G^{\prime} \in \Lambda(g, \rho)$ such that $G \cap G^{\prime} \neq \emptyset$. Let $W_{1}$ be the size of the maximum independent set in $\mathcal{G}$. Let $W_{2}$ be the size of the maximum induced matching in $\mathcal{G}$. Let $W_{3}$ be the maximum degree of $\mathcal{G}$. Then by a simple argument, one gets that the number of vertices in $\mathcal{G}$, which is $|\Lambda(g, \rho)|$, is at most $W_{1}+2 W_{2} W_{3}$. (Indeed, we can partition the set of vertices of $\mathcal{G}$ to those that are adjacent to a vertex in some fixed induced matching of largest size, and to those that are not. The first part of the partition trivially has size at most $2 W_{2} W_{3}$. The second part of the partition is an independent set and so has size at most $W_{1}$.) Hence, in order to prove (3), it is enough to show that $W_{1}$ and $W_{2} W_{3}$ are sufficiently small with probability $1-n^{-\omega(1)}$. Specifically we will show the following:

$$
\begin{aligned}
\operatorname{Pr}\left[W_{1} \geq \lambda k^{e_{H}-1}+k^{e_{H} / 2-1 / 3} / 3\right] & \leq n^{-\omega(1)} \\
\operatorname{Pr}\left[W_{2} \geq \ln n\right] & \leq n^{-\omega(1)} \\
\operatorname{Pr}\left[W_{3} \geq \ln n\right] & \leq n^{-\omega(1)}
\end{aligned}
$$

Note that by the argument above, (6-8) imply via the union bound that with probability $1-n^{-\omega(1)},|\Lambda(g, \rho)| \leq \lambda k^{e_{H}-1}+k^{e_{H} / 2-1 / 3} / 2$, so it remains to prove $(6-8)$.

We start by proving (8). Since there are at most $n^{v_{H}-2}$ subgraphs in $\Lambda(g, 1)$, it is enough to fix $G \in \Lambda(g, 1)$ and prove that, with probability $1-n^{-\omega(1)}$, either $G$ is not a vertex in $\mathcal{G}$, or $G$ has degree less than $\ln n$ in $\mathcal{G}$. So let us fix $G \in \Lambda(g, 1)$. For $t \geq 0$, we say that a sequence $S=\left(G_{j}\right)_{j=0}^{t}$ of subgraphs $G_{j} \in \Lambda(g, 1)$ is a $(G, t)$-star, if $G_{0}=G$ and if for every $j \geq 1$ the following two conditions hold: (i) $G_{0} \cap G_{j} \neq \emptyset$, and (ii) $G_{j}$ has an edge which do not belong to any $G_{j^{\prime}}, j^{\prime}<j$. We say that $\mathbb{G}(n, \rho)$ contains a $(G, t)$-star $S$ and write $\{S \subseteq \mathbb{G}(n, \rho)\}$ for that event, if for every subgraph $G_{j} \in S, G_{j} \subseteq \mathbb{G}(n, \rho)$. We first observe that if no $(G, t)$-star is contained in $\mathbb{G}(n, \rho)$, then either $G$ is not a vertex of $\mathcal{G}$, or the degree of $G$ in $\mathcal{G}$ is at most $O\left(t^{e_{H}}\right)$. Indeed, if $t=0$ then clearly $G$ is not a vertex in $\mathcal{G}$; So assume $t \geq 1$ and and let $S$ be a maximal $\left(G, t^{\prime}\right)$-star that is contained in $\mathbb{G}(n, \rho)$ (here maximal means that $\mathbb{G}(n, \rho)$ contains no $\left(G, t^{\prime}+1\right)$-star). Then by maximality of $S$, any vertex that is adjacent to $G$ in $\mathcal{G}$ is either in the sequence $S$, or is fully contained in $E(S)$, where $E(S)$ denotes the set of all edges of the subgraphs in $S$. Since $|E(S)|=O(t)$, it then follows trivially that the number of vertices adjacent to $G$ in $\mathcal{G}$ is at most $O\left(t^{e_{H}}\right)$. Hence, in order to prove (8) it remains to show that with probability $1-n^{-\omega(1)}, \mathbb{G}(n, \rho)$ contains no $(G,\lfloor\ln \ln n\rfloor)$-star, say. For brevity, below we assume that $\ln \ln n$ is an integer.

Let $Z_{t}$ denote the number of $(G, t)$-stars that are contained in $\mathbb{G}(n, \rho)$, where $G$ is the subgraph fixed above. Since the probability that $\mathbb{G}(n, \rho)$ contains a $(G, t)$-star is at most $\mathbb{E}\left[Z_{t}\right]$, it is enough to show that for $t=\ln \ln n, \mathbb{E}\left[Z_{t}\right]$ is upper bounded by $n^{-\omega(1)}$. Denote by $\operatorname{Star}_{t}$ the set of all $(G, t)$-stars. For $S=\left(G_{j}\right)_{j=0}^{t-1} \in \operatorname{Star}_{t-1}$, denote by $\mathcal{E}_{t}(S)$ the set of 
all $G_{t} \in \Lambda(g, 1)$ such that $\left(S, G_{t}\right):=\left(G_{j}\right)_{j=0}^{t} \in \operatorname{Star}_{t}$. Then for $t \geq 1$,

$$
\begin{aligned}
\mathbb{E}\left[Z_{t}\right] & =\sum_{S \in \text { Star }_{t}} \operatorname{Pr}[S \subseteq \mathbb{G}(n, \rho)] \\
& =\sum_{S \in \text { Star }_{t-1}} \operatorname{Pr}[S \subseteq \mathbb{G}(n, \rho)] \cdot \sum_{G_{t} \in \mathcal{E}_{t}(S)} \operatorname{Pr}\left[G_{t} \subseteq \mathbb{G}(n, \rho) \mid S \subseteq \mathbb{G}(n, \rho)\right] .
\end{aligned}
$$

Take $t \in[\ln \ln n]$ and fix $S \in \operatorname{Star}_{t-1}$. Note that the number of subgraphs $G_{t} \in \mathcal{E}_{t}(S)$ such that $\left(G_{t} \cup\{g\}\right) \cap(E(S) \cup\{g\})$ is isomorphic to $F \subseteq H$ is at most $O\left(n^{v_{H}-v_{F}} t^{v_{H}-2}\right)$, which for our choice of $t$ is at most $n^{v_{H}-v_{F}+o(1)}$. Moreover, for such subgraphs $G_{t}$, we have that the probability of $\left\{G_{t} \subseteq \mathbb{G}(n, \rho) \mid S \subseteq \mathbb{G}(n, \rho)\right\}$ is exactly $\rho^{e_{H}-e_{F}}$. Also note that for every $G_{t} \in \mathcal{E}_{t}(S),\left(G_{t} \cup\{g\}\right) \cap(E(S) \cup\{g\})$ is isomorphic to some $F \subsetneq H$ with $v_{F} \geq 3$. Hence, letting $\sum_{F}$ be the sum over all $F \subsetneq H$ with $v_{F} \geq 3$, we have for our choice of $t$ that there exists $\varepsilon_{H}>0$ such that:

$$
\sum_{G_{t} \in \mathcal{E}_{t}(S)} \operatorname{Pr}\left[G_{t} \subseteq \mathbb{G}(n, \rho) \mid S \subseteq \mathbb{G}(n, \rho)\right] \leq \sum_{F} O\left(n^{v_{H}-v_{F}+o(1)} \rho^{e_{H}-e_{F}}\right) \leq n^{-\varepsilon_{H}+o(1)},
$$

where the last inequality is from Fact 3.1. Hence for $t \in[\ln \ln n]$,

$$
\mathbb{E}\left[Z_{t}\right] \leq \mathbb{E}\left[Z_{t-1}\right] \cdot n^{-\varepsilon_{H}+o(1)}
$$

As there is only one $(G, 0)$-star, $\mathbb{E}\left[Z_{0}\right] \leq 1$. Hence we conclude from (9) that $\mathbb{E}\left[Z_{t}\right] \leq$ $n^{-\left(\varepsilon_{H}-o(1)\right) t}$ for all $t \in[\ln \ln n]$. Thus, for $t=\ln \ln n, \mathbb{E}\left[Z_{t}\right]=n^{-\omega(1)}$. This concludes the proof of (8).

Next we prove (7). Let $Y_{t}$ denote the number of induced matchings of size $t$ in $\mathcal{G}$. Since the expectation of $Y_{t}$ is an upper bound on the probability that there exists an induced matching of size $t$ in $\mathcal{G}$, in order to prove (7) it is enough to show that for $t=\lfloor\ln n\rfloor$, $\mathbb{E}\left[Y_{t}\right]=n^{-\omega(1)}$. Let $\mathcal{G}^{*}$ be the graph whose vertex set is $\Lambda(g, 1)$ and whose edge set consists of all pairs of distinct vertices $G, G^{\prime}$ such that $G \cap G^{\prime} \neq \emptyset$. Let $\operatorname{Match}_{t}$ be the collection of all induced matchings of size $t$ in $\mathcal{G}^{*}$. For $M \in \operatorname{Match}_{t-1}$, let $\mathcal{E}_{t}(M)$ denote the set of all edges $\left(G_{t}, G_{t}^{\prime}\right)$ in $\mathcal{G}^{*}$ such that $M \cup\left\{\left(G_{t}, G_{t}^{\prime}\right)\right\} \in \operatorname{Match}_{t}$. The number of edges $\left(G_{t}, G_{t}^{\prime}\right) \in \mathcal{E}_{t}(M)$ such that $\left(G_{t} \cup\{g\}\right) \cap\left(G_{t}^{\prime} \cup\{g\}\right)$ is isomorphic to $F \subseteq H$ is at most $O\left(n^{2 v_{H}-2-v_{F}}\right)$; Moreover, for such an edge $\left(G_{t}, G_{t}^{\prime}\right)$, the probability of the event $\left\{G_{t}, G_{t}^{\prime} \subseteq \mathbb{G}(n, \rho)\right\}$ is $\rho^{2 e_{H}-1-e_{F}}$, even conditioning on the event $\left\{G, G^{\prime} \subseteq \mathbb{G}(n, \rho):\left(G, G^{\prime}\right) \in M\right\}$. Trivially, for an edge $\left(G_{t}, G_{t}^{\prime}\right) \in \mathcal{E}_{t}(M)$, we have that $\left(G_{t} \cup\{g\}\right) \cap\left(G_{t}^{\prime} \cup\{g\}\right)$ is isomorphic to a proper subgraph $F$ of $H$ over at least 3 vertices. Thus, if $\sum_{F}$ is the sum over all $F \subsetneq H$ with 
$v_{F} \geq 3$, we have for $t \geq 1$,

$$
\begin{aligned}
\mathbb{E}\left[Y_{t}\right]= & \sum_{M \in \operatorname{Match}_{t}} \operatorname{Pr}\left[G, G^{\prime} \subseteq \mathbb{G}(n, \rho):\left(G, G^{\prime}\right) \in M\right] \\
\leq & \sum_{M \in \operatorname{Match}_{t-1}} \operatorname{Pr}\left[G, G^{\prime} \subseteq \mathbb{G}(n, \rho):\left(G, G^{\prime}\right) \in M\right] . \\
& \sum_{\left(G_{t}, G_{t}^{\prime}\right) \in \mathcal{E}_{t}(M)} \operatorname{Pr}\left[G_{t}, G_{t}^{\prime} \subseteq \mathbb{G}(n, \rho) \mid G, G^{\prime} \subseteq \mathbb{G}(n, \rho):\left(G, G^{\prime}\right) \in M\right] \\
\leq & \mathbb{E}\left[Y_{t-1}\right] \cdot \sum_{F} O\left(n^{2 v_{H}-2-v_{F}} \rho^{2 e_{H}-1-e_{F}}\right) \leq \mathbb{E}\left[Y_{t-1}\right] \cdot n^{-\varepsilon_{H}+o(1)},
\end{aligned}
$$

where the last inequality follows from the fact that $n^{v_{H}-2} \rho^{e_{H}-1}=k^{e_{H}-1}=n^{o(1)}$ and from Fact 3.1 , so $\varepsilon_{H}>0$ depends only on $H$. Since trivially $\mathbb{E}\left[Y_{0}\right]=1$, from (10) we can conclude that $\mathbb{E}\left[Y_{t}\right]=n^{-\omega(1)}$, for $t=\lfloor\ln n\rfloor$. This gives us $(7)$.

Lastly, we prove (6). For this we use the next tail bound due to Spencer [11] (See also [7], Lemma 2.46). If $X$ denotes the number of vertices in $\mathcal{G}$ then

$$
\operatorname{Pr}\left[W_{1} \geq \mathbb{E}[X]+t\right] \leq \exp \left(-\frac{t^{2}}{2(\mathbb{E}[X]+t / 3)}\right)
$$

Using the fact that $\mathbb{E}[X]=\lambda k^{e_{H}-1}$, taking $t=k^{e_{H} / 2-1 / 3} / 3$, using the fact that $k^{1 / 3}=$ $\omega(\ln n)$, we can conclude from (11) the validity of $(6)$.

\subsection{Bounding $\operatorname{Pr}\left[E_{2}(f)\right]$}

For the rest of this section we fix an edge $f \in K_{n}$. We show that $E_{2}(f)$ occurs with probability $1-o(1)$.

Definition 4 (bad sequence). Let $S=\left(G_{1}, G_{2}, \ldots, G_{d}\right)$ be a sequence of subgraphs of $K_{n}$ with $2 \leq d \leq 2 D$. We say that $S$ is a bad sequence if the following three items hold simultaneously:

1. For all $j \in[d], G_{j} \in \Lambda(g, 1)$ for some edge $g \in\{f\} \cup \bigcup_{i<j} G_{i}$.

2. For all $j \in[d-1], G_{j}$ shares exactly 2 vertices and 0 edges with $\{f\} \cup \bigcup_{i<j} G_{i}$.

3. $G_{d}$ shares at least 3 vertices and at most $e_{H}-2$ edges with $\{f\} \cup \bigcup_{i<d} G_{i}$.

For a bad sequence $S=\left(G_{1}, G_{2}, \ldots, G_{d}\right)$, write $\{S \subseteq \mathbb{G}(n, \rho)\}$ for the event that for every $j \in[d],\left\{G_{j} \subseteq \mathbb{G}(n, \rho)\right\}$ occurs. Let $E_{3}$ be the event that for all bad sequences $S$, $\{S \subseteq \mathbb{G}(n, \rho)\}$ does not occur. The next two propositions imply the required lower bound of $1-o(1)$ on the probability of $E_{2}(f)$, by first showing that $E_{3}$ implies $E_{2}(f)$ and then showing that the probability of $E_{3}$ is $1-o(1)$.

Proposition 3.2. $E_{3}$ implies $E_{2}(f)$. 
Proof. Assume $E_{3}$ occurs. Then for every bad sequence $S,\{S \subseteq \mathbb{G}(n, \rho)\}$ does not occur. To prove the assertion in the proposition, we need to show that the tree $T_{f, D}$ defined in Definition 2 is a good tree. To do this, we need to show that $T_{f, D}$ satisfies properties $\mathrm{P} 1$, P2 and P3, as given in Definition 3. We start the proof by showing, using the following claim, the $T_{f, D}$ satisfies property $\mathrm{P} 1$ (and part of property $\mathrm{P} 2$ ).

Claim 3.3. For $d \in[D]$, let $P=\left(v_{0}, u_{1}, v_{1}, \ldots, u_{d-1}, v_{d-1}, u_{d}, v_{d}\right)$ be a directed path in $T_{f, d}$ from the root $v_{0}$ to a leaf $v_{d}$. Let $G_{j}$ be the label of node $u_{j}$ and let $g_{j}$ be the label of node $v_{j}$ (so that $g_{0}=f$ ). Then (i) $G_{d} \cap\{f\}=\emptyset$, and (ii) $G_{d} \cap G_{i}=\emptyset$, for every $0 \leq i \leq d-1$.

Proof. The proof is by induction on $d$. Clearly, the claim is valid for $d=1$, as by definition, any subgraph in $\Lambda(f, \rho)$ does not contain the edge $f$. Let $d \geq 2, d \in[D]$ and assume the claim holds for $d-1$. We prove the claim for $d$. Let $S=\left(G_{1}, G_{2}, \ldots, G_{d}\right)$ be the sequence of the labels of the nodes $u_{i}, i \in[d]$, along the path $P$. Assume for the sake of contradiction that $G_{d}$ shares some edge with $\{f\} \cup \bigcup_{i<d} G_{i}$. We shall reach a contradiction by showing that either $S$ is a bad sequence (this contradicts the occurrence of $E_{3}$ ), or $P$ is not a directed path in $T_{f, d}$.

Note first that from the induction hypothesis we have that for every $j \in[d-1]$, $G_{j}$ shares no edge with $\{f\} \cup \bigcup_{i<j} G_{i}$. We claim that this implies also that for every $j \in[d-1], G_{j}$ shares exactly 2 vertices with $\{f\} \cup \bigcup_{i<j} G_{i}$. Indeed, for $d=2$ this claim is true by definition. If the claim is not true for $d \geq 3$ then we have for some $j \in[d-1]$, $j \geq 2$, that $\left(G_{1}, G_{2}, \ldots, G_{j}\right)$ is a bad sequence, contradicting $E_{3}$.

Now, by assumption, $G_{d}$ shares some edge with $\{f\} \cup \bigcup_{i<d} G_{i}$. If we also have that $G_{d}$ shares at most $e_{H}-2$ edges with $\{f\} \cup \bigcup_{i<d} G_{i}$ then by the observation made in the previous paragraph we are done, since this implies that $S$ is a bad sequence. Therefore, we can assume for the rest of the proof that $G_{d}$ shares all of its $e_{H}-1$ edges with $\{f\} \cup \bigcup_{i<d} G_{i}$. We shall reach a contradiction by showing that $P$ is not a directed path in $T_{f, d}$.

Write $g_{d-1}=\{a, b\}$ and $g_{d-2}=\{x, y\}$ and recall that $G_{d-1} \in \Lambda\left(g_{d-2}, \rho\right)$ and $G_{d} \in$ $\Lambda\left(g_{d-1}, \rho\right)$. Observe that $g_{d-2} \neq g_{d-1}$. Hence, we may assume without loss of generality that $a \notin\{x, y\}$. Note that $a$ is a vertex of both $G_{d}$ and $G_{d-1}$. Now, a key observation is that any edge in $G_{d}$ that is adjacent to a must belong also to $G_{d-1}$, for otherwise, the subgraph $G_{d-1}$ will share 3 vertices $\left(x, y\right.$ and $a$ ) with $\{f\} \cup \bigcup_{i<d-1} G_{i}$-and that contradicts the fact established above. More generally and for the same reason, if $a^{\prime} \notin\{x, y\}$ is a vertex of both $G_{d}$ and $G_{d-1}$, then any edge adjacent to $a^{\prime}$ in $G_{d}$ must also belong to $G_{d-1}$. With that key observation at hand, we conclude the proof by reaching a contradiction for every possible choice for the graph $H$.

Suppose first that $H=K_{3}$. Without loss of generality, we have $b=x$. Now, since any edge that is adjacent to $a$ in $G_{d}$ must also be an edge in $G_{d-1}$, it follows that $\{a, y\}$ is an edge in $G_{d}$. Therefore, $\{a, b=x, y\}$ is the set of vertices of $G_{d}$ and so $g_{d-2}=\{x, y\}$ is an edge in $G_{d}$. But, by Definition 2, this contradicts the assumption that $P$ is a directed path in $T_{f, d}$.

To reach a contradiction for other regular, strictly 2-balanced graphs, we need the following fact. 
Fact 3.4. Let $H$ be a regular, strictly 2-balanced graph with $v_{H} \geq 4$. Let $\{x, y\}$ be an edge in $H$ and let $a, a^{\prime} \notin\{x, y\}$ be two distinct vertices in $H$. Then there is a path from a to $a^{\prime}$ in $H$ that avoids the vertices $\{x, y\}$.

Proof. Assume for the sake of contradiction that there exist two distinct vertices $a, a^{\prime} \notin$ $\{x, y\}$ such that every path in $H$ from $a$ to $a^{\prime}$, if there exists any, must go through a vertex in $\{x, y\}$. This implies that we can write $H$ as the union of two graphs, $H_{1}$ and $H_{2}$, that share only the edge $\{x, y\}$ and the vertices $x$ and $y$, and such that $a$ is a vertex in $H_{1}$ and $a^{\prime}$ is a vertex in $H_{2}$. Note that $v_{H_{1}}, v_{H_{2}} \geq 3$ and that $H_{1}$ and $H_{2}$ are proper subgraphs of $H$. Without loss of generality, we assume that $\left(e_{H_{1}}-1\right) /\left(v_{H_{1}}-2\right) \geq\left(e_{H_{2}}-1\right) /\left(v_{H_{2}}-2\right)$. Now, by the fact that $H=H_{1} \cup H_{2}$ is strictly 2-balanced, we have $\left(e_{H}-1\right) /\left(v_{H}-2\right)>$ $\left(e_{H_{1}}-1\right) /\left(v_{H_{1}}-2\right)$. Therefore, from the last two inequalities we deduce that

$$
\begin{aligned}
\left(e_{H_{1}}-1\right)\left(v_{H}-2\right) & <\left(v_{H_{1}}-2\right)\left(e_{H}-1\right), \quad \text { and } \\
-\left(e_{H_{1}}-1\right)\left(v_{H_{2}}-2\right) & \leq-\left(v_{H_{1}}-2\right)\left(e_{H_{2}}-1\right) .
\end{aligned}
$$

Hence,

$$
\left(e_{H_{1}}-1\right)\left(v_{H}-v_{H_{2}}\right)<\left(v_{H_{1}}-2\right)\left(e_{H}-e_{H_{2}}\right) .
$$

Applying the facts that $v_{H}=v_{H_{1}}+v_{H_{2}}-2$ and $e_{H}=e_{H_{1}}+e_{H_{2}}-1$ to the last inequality, we get $\left(e_{H_{1}}-1\right)\left(v_{H_{1}}-2\right)<\left(v_{H_{1}}-2\right)\left(e_{H_{1}}-1\right)$, which is a clear contradiction.

Suppose now that $H$ is regular, strictly 2-balanced and $v_{H} \geq 4$. We use Fact 3.4 in order to generalize the argument for $K_{3}$ given above. Define $G_{d}^{+}:=G_{d} \cup\left\{g_{d-1}\right\}$. We will show below that $G_{d-1} \subseteq G_{d}^{+}$. Since $H$ is regular, this implies that $g_{d-2} \in G_{d}^{+}$. Since $G_{d}^{+}=G_{d} \cup\left\{g_{d-1}\right\}$ and $g_{d-1} \neq g_{d-2}$ it then follows that $g_{d-2} \in G_{d}$. This, by Definition 2, contradicts the assumption that $P$ is a directed path in $T_{f, d}$. It thus remains to show $G_{d-1} \subseteq G_{d}^{+}$. Since every edge in $G_{d-1}$ is adjacent to some vertex $a^{\prime} \notin\{x, y\}$, it is enough to show that for every vertex $a^{\prime} \notin\{x, y\}$ of $G_{d-1}$, any edge adjacent to $a^{\prime}$ in $G_{d-1}$ is an edge in $G_{d}^{+}$.

Let $a^{\prime} \notin\{x, y\}$ be an arbitrary vertex of $G_{d-1}$. By Fact 3.4 there exists in $G_{d-1}$ a path $\left(a_{0}=a, a_{1}, a_{2}, \ldots, a_{l}=a^{\prime}\right)$ of length $l \geq 0$ from $a$ to $a^{\prime}$, that avoids the vertices $\{x, y\}$. We first claim that $a_{i}$ is a vertex of $G_{d}^{+}$for every $0 \leq i \leq l$. Indeed, the claim is trivially true for $i=0$, as $a_{0}=a$ is a vertex of $G_{d} \subset G_{d}^{+}$. Assume that for $0 \leq i-1<l, a_{i-1}$ is a vertex of $G_{d}^{+}$. Note that in that case, $a_{i-1}$ is a vertex of both $G_{d}$ and $G_{d-1}$ and that $a_{i-1} \notin\{x, y\}$. Then by the key observation made above, every edge that is adjacent to $a_{i-1}$ in $G_{d}$ is an edge in $G_{d-1}$. Also, we have $g_{d-1} \in G_{d-1}$. Therefore, every edge that is adjacent to $a_{i-1}$ in $G_{d}^{+}$is an edge in $G_{d-1}$. This, together with the facts that $H$ is regular and $a_{i-1} \notin\{x, y\}$ implies that every edge that is adjacent to $a_{i-1}$ in $G_{d-1}$ is an edge in $G_{d}^{+}$. Therefore, since $\left\{a_{i-1}, a_{i}\right\}$ is an edge in $G_{d-1}$, we get as needed that $a_{i}$ is a vertex of $G_{d}^{+}$.

Now that we have found that $a^{\prime}$ is a vertex of both $G_{d}^{+}$and $G_{d-1}$, using the fact that $a^{\prime} \notin\{x, y\}$, we again have by the key observation above that every edge that is adjacent to $a^{\prime}$ in $G_{d}$ is an edge in $G_{d-1}$. Since $g_{d-1} \in G_{d-1}$ we thus have that every edge 
that is adjacent to $a^{\prime}$ in $G_{d}^{+}$is an edge in $G_{d-1}$. Therefore, by regularity of $H$ and since $a^{\prime} \notin\{x, y\}$, we have that every edge that is adjacent to $a^{\prime}$ in $G_{d-1}$ is an edge in $G_{d}^{+}$. This completes the proof of the claim.

Claim 3.3 gives us property P1 (and part of property P2). We now prove that property P2 holds as well.

Claim 3.5. For any $d \in[D]$, property P® holds for $T_{f, d}$.

Proof. The proof is by induction on $d$. To see that property P2 holds for the base case, $d=1$, let $\left(v_{0}, u_{1}\right)$ and $\left(v_{0}, u_{1}^{\prime}\right)$ be two different paths in $T_{f, 1}$ so that $u_{1} \neq u_{1}^{\prime}$. Let $G_{1}$ and $G_{2}$ be the labels of the nodes $u_{1}$ and $u_{1}^{\prime}$, and assume for the sake of contradiction that $G_{1} \cap G_{2} \neq \emptyset$. Then clearly $G_{2}$ shares an edge with $\{f\} \cup G_{1}$. Since $G_{1}$ and $G_{2}$ are distinct subgraphs (or else $u_{1}=u_{1}^{\prime}$ ), and using the fact that $G_{2} \in \Lambda(f, \rho)$ and so $G_{2} \cap\{f\}=\emptyset$, we also have that $G_{2}$ shares at most $e_{H}-2$ edges with $\{f\} \cup G_{1}$. Hence, by definition, $\left(G_{1}, G_{2}\right)$ is a bad sequence. This contradicts $E_{3}$ and so property $\mathrm{P} 2$ holds for $T_{f, 1}$.

Let $d \in[D], d \geq 2$ and assume the claim holds for $d-1$. We prove that $\mathrm{P} 2$ holds for $T_{f, d}$. Let $P=\left(v_{0}, u_{1}, v_{1}, \ldots, v_{d-1}, u_{d}, v_{d}\right)$ be a path in $T_{f, d}$ from the root $v_{0}$ to a leaf. For $j \in[d]$, let $G_{j}$ be the label of the node $u_{j}$. Note that by the induction hypothesis and by Claim 3.3, in order to prove the claim for $T_{f, d}$, it suffices to show that $G_{d}$ does not share an edge with the label of any node $u \notin\left\{u_{1}, u_{2}, \ldots, u_{d}\right\}$ in $T_{f, d}$, where $u$ is at odd distance from $v_{0}$. Assume for the sake of contradiction that for some $v_{i}, 0 \leq i \leq d-1$, and some $l \geq 1$, there exists a path $P^{\prime}=\left(v_{i}, u_{1}^{\prime}, v_{1}^{\prime}, \ldots, v_{l-1}^{\prime}, u_{l}^{\prime}, v_{l}^{\prime}\right)$ in $T_{f, d}$ such that $u_{1} \neq u_{1}^{\prime}$ (and so $\left.u_{l}^{\prime} \notin\left\{u_{1}, u_{2}, \ldots, u_{d}\right\}\right)$ and yet the labels of $u_{d}$ and $u_{l}^{\prime}$ have a non-empty intersection. We will reach a contradiction by constructing a bad sequence from the labels along the paths $P$ and $P^{\prime}$.

For $j \in[l]$, let $G_{j}^{\prime}$ be the label of node $u_{j}^{\prime}$. We note that by assumption, $\left|G_{d} \cap G_{l}^{\prime}\right| \geq 1$. We assume for simplicity that $l$ is minimal, in the sense that $\left|G_{d} \cap G_{j}^{\prime}\right|=0$ for every $1 \leq j \leq l-1$, or else we can shorten the path $P^{\prime}$ so as to satisfy this assumption and still construct a bad sequence and get a contradiction. We consider two cases:

- Assume $v_{l}^{\prime}$ is a node in $T_{f, d-1}$ so that $P^{\prime}$ is a path in $T_{f, d-1}$. Define $S$ to be the sequence which is the concatenation of $\left(G_{1}, G_{2}, \ldots, G_{d-1}\right)$ with $\left(G_{1}^{\prime}, G_{2}^{\prime}, \ldots, G_{l}^{\prime}\right)$. For convenience, rewrite $S=\left(F_{1}, F_{2}, \ldots, F_{l+d-1}\right)$ (here $F_{1}=G_{1}$ and $F_{l+d-1}=G_{l}^{\prime}$ ). From the induction hypothesis and claim 3.3 we have that for every $j \in[l+d-1]$, $F_{j}$ shares no edge with $\{f\} \cup \bigcup_{i<j} F_{i}$. From this, together with the occurrence of $E_{3}$ we get that for all $j \in[l+d-1], F_{j}$ shares exactly 2 vertices with $\{f\} \cup \bigcup_{i<j} F_{i}$. We now claim that the sequence $S^{\prime}=\left(F_{1}, F_{2}, \ldots, F_{l+d-1}, G_{d}\right)$ is a bad sequence. First note that by the minimality of $l, G_{d}$ shares no edge with $\bigcup_{i<l} G_{i}^{\prime}$. Also, by Claim 3.3, $G_{d}$ shares no edge with $\{f\} \cup \bigcup_{i<d} G_{i}$. In contrast, by assumption, $G_{d}$ shares at least one edge with $G_{l}^{\prime}$. Note that $S^{\prime}$ contains at most $2 D$ subgraphs. Hence, to demonstrate that $S^{\prime}$ is a bad sequence and get a contradiction it is enough to show that $G_{d}$ shares at most $e_{H}-2$ edges with $G_{l}^{\prime}$.

If $G_{d}$ shares $e_{H}-1$ edges with $G_{l}^{\prime}$ then $G_{d}=G_{l}^{\prime}$. In that case, since $H$ is regular, we would have that the label of the node $v_{d-1}$ is the same as the label of the node 
$v_{l-1}^{\prime}$. But since clearly $v_{d-1} \neq v_{l-1}^{\prime}$ (indeed, the distance of $v_{d-1}$ from $v_{0}$ is strictly larger than the distance of $v_{l-1}^{\prime}$ from $v_{0}$ ), this violates either property P2 of $T_{f, d-1}$ which holds by the induction hypothesis, or property $\mathrm{P} 1$ which we have already established. Hence $G_{d}$ shares at most $e_{H}-2$ edges with $G_{l}^{\prime}$ and we conclude that $S^{\prime}$ is a bad sequence, a contradiction to $E_{3}$.

- Assume $v_{l}^{\prime}$ is a leaf in $T_{f, d}$. Define the sequence $S=\left(F_{1}, F_{2}, \ldots, F_{l+d-1}\right)$ as in the previous item. From the induction hypothesis, Claim 3.3 and from the previous item we have that for every $j \in[l+d-1], F_{j}$ shares no edge with $\{f\} \cup \bigcup_{i<j} F_{i}$. By the occurrence of $E_{3}$ we then have that for every $j \in[l+d-1], F_{j}$ shares exactly 2 vertices with $\{f\} \cup \bigcup_{i<j} F_{i}$.

Define $S^{\prime}=\left(F_{1}, F_{2}, \ldots, F_{l+d-1}, G_{d}\right)$ as in the previous item. We again claim that $S^{\prime}$ is a bad sequence. To verify this claim, first note that by Claim 3.3, $G_{d}$ shares no no edge with $\{f\} \cup \bigcup_{i<d} G_{i}$. By the minimality of $l, G_{d}$ shares no edge with $\bigcup_{i<l} G_{i}^{\prime}$. Also, there are at most $2 D$ subgraphs in $S^{\prime}$ and by assumption, $G_{d}$ shares some edge with $G_{l}^{\prime}$. Hence, to conclude that $S^{\prime}$ is a bad sequence, it is enough to show that $G_{d}$ shares at most $e_{H}-2$ edges with $G_{l}^{\prime}$. We have two cases.

1. Suppose $v_{d-1}=v_{l-1}^{\prime}$. Since $u_{d} \neq u_{l}^{\prime}$ we have by definition that $G_{d}$ shares at most $e_{H}-2$ edges with $G_{l}^{\prime}$.

2. Suppose $v_{d-1} \neq v_{l-1}^{\prime}$. If $G_{d}$ shares all $e_{H}-1$ edges with $G_{l}^{\prime}$ then since $H$ is regular, we have that the labels of $v_{d-1}$ and $v_{l-1}^{\prime}$ are the same. This in turn implies that the parents of $v_{d-1}$ and $v_{l-1}$ are distinct and their labels share an edge. This is a contradiction to the induction hypothesis. Hence, as needed, $G_{d}$ shares at most $e_{H}-2$ edges with $G_{l}^{\prime}$.

We conclude that property $\mathrm{P} 2$ holds for $T_{f, d}$.

To conclude the proof we argue that property P3 holds. Let $\left(v_{0}, u_{1}, v_{1}, \ldots, v_{d-1}, u_{d}, v_{d}\right)$ be a path in $T_{f, D}$, starting from the root $v_{0}$. Let $G_{j}$ be the label of node $u_{j}$ and let $g_{j}$ be the label of node $v_{j}$. We need to show that the number of nodes adjacent to $v_{d-1}$ in $T_{f, D}$ is $\left|\Lambda\left(g_{d-1}, \rho\right)\right|-O(1)$. The claim is trivially true for $d=1$, since any subgraph in $\Lambda(f, \rho)$ is a label of a node adjacent to the root of $T_{f, D}$. For $d \geq 2$, we recall that by definition, the nodes that are adjacent to $v_{d-1}$ in $T_{f, D}$ are those nodes whose labels are in $\left\{G_{d} \in \Lambda\left(g_{d-1}, \rho\right): g_{d-2} \notin G_{d}\right\}$. Reflecting on the proof of Claim 3.3, we see that assuming $E_{3}$, if $g_{d-2} \in G_{d} \in \Lambda\left(g_{d-1}, \rho\right)$, then the set of vertices of $G_{d}$ is the same as that of $G_{d-1}$. This immediately implies that $\left|\left\{G_{d} \in \Lambda\left(g_{d-1}, \rho\right): g_{d-2} \in G_{d}\right\}\right|=O(1)$. This gives us property $\mathrm{P} 3$. With that, we conclude the proof.

Proposition 3.6. $\operatorname{Pr}\left[E_{3}\right]=1-o(1)$.

Proof. Let $Z$ be the random variable counting the number of bad sequences $S$ for which $\{S \subseteq \mathbb{G}(n, \rho)\}$ occurs. Since the probability that $\{S \subseteq \mathbb{G}(n, \rho)\}$ occurs for some bad sequence $S$ is at most $\mathbb{E}[Z]$, showing that $\mathbb{E}[Z]=o(1)$ would imply the proposition. 
For $d \geq 2$, let $\mathrm{Seq}_{d}$ denote the collection of all bad sequences of length $d$. Then

$$
\mathbb{E}[Z]=\sum_{2 \leq d \leq 2 D} \sum_{S_{d} \in \mathrm{Seq}_{d}} \operatorname{Pr}\left[S_{d} \subseteq \mathbb{G}(n, \rho)\right] .
$$

Below we show that for every $d$ satisfying $2 \leq d \leq 2 D$,

$$
\sum_{S_{d} \in \mathrm{Seq}_{d}} \operatorname{Pr}\left[S_{d} \subseteq \mathbb{G}(n, \rho)\right] \leq n^{-\varepsilon_{H}+o(1)}
$$

where $\varepsilon_{H}>0$ is the constant provided in Fact 3.1. From (12) and (13) and since $2 D=$ $n^{o(1)}$, we get that $\mathbb{E}[Z] \leq n^{-\varepsilon_{H}+o(1)}=o(1)$ as required. Hence it remains to prove (13).

Fix for the rest of the proof $d$ satisfying $2 \leq d \leq 2 D$. In order to prove (13), it would be convenient to partition the set $\mathrm{Seq}_{d}$ to two parts and then upper bound the sum in (13) for each of the two parts of the partition. Let $\mathrm{Seq}_{d, 1}$ be the set of all sequences $S=\left(G_{i}\right)_{i=1}^{d} \in \operatorname{Seq}_{d}$ for which $G_{d}$ shares no edge (and at least 3 vertices) with $\{f\} \cup \bigcup_{i<d} G_{i}$. Let $\mathrm{Seq}_{d, 2}$ be the set of all sequences $S=\left(G_{i}\right)_{i=1}^{d} \in \operatorname{Seq}_{d}$ for which $G_{d}$ shares at least 1 edge (and at most $e_{H}-2$ edges) with $\{f\} \cup \bigcup_{i<d} G_{i}$. It would be useful to further classify those members of $\operatorname{Seq}_{d, 2}$ as follows. Suppose that $S=\left(G_{i}\right)_{i=1}^{d} \in \mathrm{Seq}_{d, 2}$. Let $g$ be the unique edge in $\{f\} \cup \bigcup_{i<d} G_{i}$ such that $G_{d} \in \Lambda(g, 1)$. (The edge $g$ is unique, since $H$ is regular.) Define

$$
H_{S}:=\{g\} \cup\left(G_{d} \cap\left(\{f\} \cup \bigcup_{i<d} G_{i}\right)\right) .
$$

Then we say that $S$ is an $F$-type if $H_{S}$ is isomorphic to $F$. Note that if $S$ is an $F$-type then $F$ is a proper subgraph of $H$ with $v_{F} \geq 3$. We are now ready to upper bound the sum in (13) for the two parts of the partition of $\mathrm{Seq}_{d}$.

We start by giving an upper bound on the sum in (13) when the sum ranges over all $S \in \mathrm{Seq}_{d, 1}$. First, we upper bound the size of $\mathrm{Seq}_{d, 1}$. To do so, we construct a bad sequence $S=\left(G_{i}\right)_{i=1}^{d} \in \operatorname{Seq}_{d, 1}$ iteratively. Assume we have already chosen the first $j-1$ subgraphs in $S$ for $j \in[d-1]$. We count the number of choices for $G_{j}$ : There are $O(d)$ possible choices for an edge $g \in\{f\} \cup \bigcup_{i<j} G_{i}$ for which $G_{j}$ is in $\Lambda(g, 1)$; There are at most $n^{v_{H}-2}$ choices for the vertices of $G_{j}$; There are $O(1)$ choices for the edges of $G_{j}$ given that we have already fixed its $v_{H}$ vertices. In total, the number of choices for $G_{j}$ is at most $O\left(d \cdot n^{v_{H}-2}\right)=n^{v_{H}-2+O((\ln \ln n) / \ln n)}$. Assume we have already chosen the first $d-1$ subgraphs in $S$. We count the number of choices for $G_{d}$ : There are $O(d)$ possible choices for an edge $g \in\{f\} \cup \bigcup_{i<d} G_{i}$ for which $G_{d}$ is in $\Lambda(g, 1)$; There are at most $O\left(n^{v_{H}-3} \cdot d^{v_{H}}\right)$ choices for the vertices of $G_{d}$, which follows from the fact that $G_{d}$ shares at least 3 vertices with $\{f\} \cup \bigcup_{i<d} G_{i}$; There are $O(1)$ choices for the edges of $G_{d}$, given that we have fixed the $v_{H}$ vertices of $G_{d}$. In total, the number of choices for $G_{d}$ is at most $O\left(d \cdot n^{v_{H}-3} \cdot d^{v_{H}}\right)=n^{v_{H}-3+o(1)}$. From the above we conclude that the number of bad sequences $S \in \mathrm{Seq}_{d, 1}$ is at most $n^{d\left(v_{H}-2\right)-1+o(1)}$. Now, it is easy to see that if $S \in \mathrm{Seq}_{d, 1}$ then the probability of $\{S \subseteq \mathbb{G}(n, \rho)\}$ is $\rho^{d\left(e_{H}-1\right)}$. Thus, recalling Definition 1, we conclude 
that

$$
\begin{aligned}
\sum_{S \in \operatorname{Seq}_{d, 1}} \operatorname{Pr}[S \subseteq \mathbb{G}(n, \rho)] & \leq n^{d\left(v_{H}-2\right)-1+o(1)} \rho^{d\left(e_{H}-1\right)} \\
& \leq k^{d\left(e_{H}-1\right)} n^{-1+o(1)} \\
& =n^{-1+o(1)}
\end{aligned}
$$

We now upper bound the sum in (13) when the sum ranges over all $S \in \mathrm{Seq}_{d, 2}$. We first upper bound the number of $F$-type bad sequences $S \in \operatorname{Seq}_{d, 2}$, for some $F \subsetneq H$, $v_{F} \geq 3$. As before, we construct such a bad sequence $S=\left(G_{i}\right)_{i=1}^{d}$ iteratively. Assume we have already chosen the first $j-1$ subgraphs in $S$, for $j \in[d-1]$. Then the number of choices for $G_{j}$ is, as before, $n^{v_{H}-2+O((\ln \ln n) / \ln n)}$. Assume we have already chosen the first $d-1$ subgraphs in $S$. We count the number of choices for $G_{d}$ : There are $O(d)$ choices for an edge $g \in\{f\} \cup \bigcup_{i<d} G_{i}$ such that $G_{d} \in \Lambda(g, 1)$; There are at most $O\left(n^{v_{H}-v_{F}} \cdot d^{v_{F}}\right)$ choices for the vertices of $G_{d}$; There are $O(1)$ choices for the edges of $G_{d}$, given that we have fixed its edges. In total, the number of choices for $G_{d}$ is $O\left(d \cdot n^{v_{H}-v_{F}} \cdot d^{v_{F}}\right)=$ $n^{v_{H}-v_{F}+o(1)}$. Hence, we conclude that the number of $F$-type bad sequences $S \in \mathrm{Seq}_{d, 2}$ is $n^{(d-1)\left(v_{H}-2\right)+v_{H}-v_{F}+o(1)}$. For such a sequence $S$, it can be verified that the probability of $\{S \subseteq \mathbb{G}(n, \rho)\}$ is $\rho^{(d-1)\left(e_{H}-1\right)+e_{H}-e_{F}}$. Hence, taking $\sum_{F}$ to be the sum over all $F \subsetneq H$ with $v_{F} \geq 3$ and using Fact 3.1, we have

$$
\begin{aligned}
\sum_{S \in \operatorname{Seq}_{d, 2}} \operatorname{Pr}[S \subseteq \mathbb{G}(n, \rho)] & \leq \sum_{F} n^{(d-1)\left(v_{H}-2\right)+v_{H}-v_{F}+o(1)} \rho^{(d-1)\left(e_{H}-1\right)+e_{H}-e_{F}} \\
& \leq \sum_{F} k^{(d-1)\left(e_{H}-1\right)} n^{-\varepsilon_{H}+o(1)} \\
& =\sum_{F} n^{-\varepsilon_{H}+o(1)} .
\end{aligned}
$$

Since $\sum_{F} 1=O(1)$, we conclude from (14) and (15) the validity of (13). This completes the proof.

\section{Proof of Lemma 2.2}

In order to prove Lemma 2.2, let us fix an edge $f \in K_{n}$ and assume everywhere throughout the section that $E_{2}(f) \cap E_{1}$ occurs. Hence, we may fix once and for the rest of this section the good tree $T=T_{f, D}$ which is guaranteed to exist by the occurrence of $E_{2}(f)$. It now suffices to lower bound the probability of $\left\{f \in \mathbb{M}_{n}(H) \mid \beta(f)<c n^{-\left(v_{H}-2\right) /\left(e_{H}-1\right)}\right\}$. It is extremely important to note, and we use this fact implicitly throughout this section, that for every edge $g$ that appears as a label of a non-root node at even height in $T$, the probability of the event $\left\{\beta(g)<\rho^{\prime}\right\}$, for $\rho^{\prime} \leq \rho$, is $\rho^{\prime} / \rho$. This is true since for such an edge $g$ we already condition on the event $\{g \in \mathbb{G}(n, \rho)\}$ and so $\beta(g)$ is uniformly distributed in $[0, \rho]$. Therefore, for example, if $g$ is a label of a non-root node at even height in $T$, then the probability of $\left\{\beta(g)<c n^{-\left(v_{H}-2\right) /\left(e_{H}-1\right)}\right\}$ is $c / k$. 
A useful convention we use throughout this section is this: Every two distinct nodes in the good tree $T$ have distinct labels and so, for the rest of this section we will refer to the nodes of $T$ by their labels. We also recall and introduce some useful notation. First recall that for every node $u$ in $T, \Gamma_{T}(u)$ denotes the set of nodes adjacent to $u$ in $T$. For simplicity, we shall replace $\Gamma_{T}(u)$ with $\Gamma(u)$ when no confusion arises. Recall also that the tree $T$ has height $2 D$. If $g$ is a node at height $2 d$ in $T$, we denote by $T_{g, d}$ the subtree of $T$ that is rooted at $g$. Lastly, if $B \subseteq[0,1]^{n}$ is any event, we denote by $\bar{B}$ the event $[0,1]^{n} \backslash B$.

The overall structure of the proof of Lemma 2.2 is this: First, in the next few paragraphs, conditioning on $\left\{\beta(f)<c n^{-\left(v_{H}-2\right) /\left(e_{H}-1\right)}\right\}$, we reduce the problem of lower bounding the probability of $\left\{f \in \mathbb{M}_{n}(H)\right\}$ to the problem of lower bounding the probability of a certain event, $\overline{B\left(T_{f, D}\right)}$ (to be defined shortly), which we define using the tree $T_{f, D}$. We are then left with the task of estimating the probability of $\overline{B\left(T_{f, D}\right)}$, a task being performed in Sections 4.1 and 4.2 .

Definition 5. Let $g$ be a node in $T$ at height $2 d$ and let $T_{g, d}$ be the subtree of $T$ rooted at g. Define

$$
B\left(T_{g, d}\right):= \begin{cases}\emptyset & \text { if } d=0, \\ \exists G^{\prime} \in \Gamma(g) . \forall g^{\prime} \in G^{\prime} .\left\{\beta\left(g^{\prime}\right)<\beta(g)\right\} \cap \overline{B\left(T_{g^{\prime}, d-1}\right)} & \text { if } 1 \leq d \leq D .\end{cases}
$$

Proposition 4.1. Let $g$ be a non-leaf node in $T$ at height $2 d$ and let $T_{g, d}$ be the subtree of $T$ rooted at $g$. If $d$ is even then $B\left(T_{g, d}\right)$ implies $\left\{g \notin \mathbb{M}_{n}(H)\right\}$.

Proof. The proof is by induction on $d$. Since $g$ is a non-leaf and $d$ is even, we start with the case $d=2$ (so the distance from $g$ to any leaf of $T_{g, d}$ is 4 ). Assume $B\left(T_{g, 2}\right)$ occurs. Then by Definition 5 there exists a graph $G^{\prime} \in \Gamma(g)$ for which the following two events occur: (i) for every $g^{\prime} \in G^{\prime},\left\{\beta\left(g^{\prime}\right)<\beta(g)\right\}$, and (ii) for every $g^{\prime} \in G^{\prime}, \overline{B\left(T_{g^{\prime}, 1}\right)}$ occurs. Now observe that in order to conclude that $\left\{g \notin \mathbb{M}_{n}(H)\right\}$ occurs, it suffices to show that $\left\{G^{\prime} \subseteq \mathbb{M}_{n}(H)\right\}$ occurs. To show the occurrence of the last event, it suffices to show that for every edge $g^{\prime} \in G^{\prime}$ and for every graph $G^{\prime \prime} \in \Lambda\left(g^{\prime}, \rho\right)$, there exists an edge $g^{\prime \prime} \in G^{\prime \prime}$ whose birthtime $\beta\left(g^{\prime \prime}\right)$ is larger than $\beta\left(g^{\prime}\right)$. Let us fix $g^{\prime} \in G^{\prime}$ and $G^{\prime \prime} \in \Lambda\left(g^{\prime}, \rho\right)$. We have two cases. If $G^{\prime \prime} \in \Gamma\left(g^{\prime}\right)$ then by the fact that $\overline{B\left(T_{g^{\prime}, 1}\right)}$ occurs, we have indeed that there exists an edge $g^{\prime \prime} \in G^{\prime \prime}$ such that $\beta\left(g^{\prime \prime}\right)>\beta\left(g^{\prime}\right)$ (here we have used the fact that with probability $\left.1, \beta\left(g^{\prime \prime}\right) \neq \beta\left(g^{\prime}\right)\right)$. If on the other hand $G^{\prime \prime} \notin \Gamma\left(g^{\prime}\right)$ then by definition of $T$, $g \in G^{\prime \prime}$. Then, by item (i) above, we have that for some $g^{\prime \prime} \in G^{\prime \prime}, \beta\left(g^{\prime \prime}\right)=\beta(g)>\beta\left(g^{\prime}\right)$. We thus conclude that $\left\{g \notin \mathbb{M}_{n}(H)\right\}$ occurs.

Assume the proposition is valid for $d-2$. We prove it for $d$, so assume that $B\left(T_{g, d}\right)$ occurs. This implies by Definition 5 that there exists a graph $G^{\prime} \in \Gamma(g)$ for which the following two events occur: (i) for every $g^{\prime} \in G^{\prime},\left\{\beta\left(g^{\prime}\right)<\beta(g)\right\}$, and (ii) for every $g^{\prime} \in G^{\prime}, \overline{B\left(T_{g^{\prime}, d-1}\right)}$ occurs. As before, to prove the proposition it suffices to show that $\left\{G^{\prime} \subseteq \mathbb{M}_{n}(H)\right\}$ occurs. The occurrence of this last event can be proved if we show that for every edge $g^{\prime} \in G^{\prime}$ and for every graph $G^{\prime \prime} \in \Lambda\left(g^{\prime}, \rho\right)$, there exists an edge $g^{\prime \prime} \in G^{\prime \prime}$ such that either $\beta\left(g^{\prime \prime}\right)>\beta\left(g^{\prime}\right)$ or $g^{\prime \prime} \notin \mathbb{M}_{n}(H)$. Fix $g^{\prime} \in G^{\prime}$ and $G^{\prime \prime} \in \Lambda\left(g^{\prime}, \rho\right)$. As before, we have two cases. Suppose first that $G^{\prime \prime} \in \Gamma\left(g^{\prime}\right)$. Then there exists an edge $g^{\prime \prime} \in G^{\prime \prime}$ such that 
either $\beta\left(g^{\prime \prime}\right)>\beta\left(g^{\prime}\right)$ or $B\left(T_{g^{\prime \prime}, d-2}\right)$ occurs. This implies by the induction hypothesis that either $\beta\left(g^{\prime \prime}\right)>\beta\left(g^{\prime}\right)$ or $g^{\prime \prime} \notin \mathbb{M}_{n}(H)$, as required. The second case is that of $G^{\prime \prime} \notin \Gamma\left(g^{\prime}\right)$. Similarly to the base case, this implies that there exists an edge $g^{\prime \prime} \in G^{\prime \prime}$ (specifically $\left.g^{\prime \prime}=g\right)$ such that $\beta\left(g^{\prime \prime}\right)>\beta\left(g^{\prime}\right)$. Hence $\left\{g \notin \mathbb{M}_{n}(H)\right\}$ occurs.

Recall Definition 1 and note that $D$ is odd. Hence, from Definition 5 and Proposition 4.1, it follows that, conditioning on $\left\{\beta(f)<c n^{-\left(v_{H}-2\right) /\left(e_{H}-1\right)}\right\}, \overline{B\left(T_{f, D}\right)}$ implies $\left\{f \in \mathbb{M}_{n}(H)\right\}$. (Indeed, note that $\Gamma(f)=\Lambda(f, \rho)$ by definition.) Hence, we get that the probability of $\left\{f \in \mathbb{M}_{n}(H) \mid \beta(f)<c n^{-\left(v_{H}-2\right) /\left(e_{H}-1\right)}\right\}$ is lower bounded by

$$
\operatorname{Pr}\left[\overline{B\left(T_{f, D}\right)} \mid \beta(f)<c n^{-\left(v_{H}-2\right) /\left(e_{H}-1\right)}\right] .
$$

In the two subsections below we lower bound (16). Specifically, we show that

$$
\operatorname{Pr}\left[\overline{B\left(T_{f, D}\right)} \mid \beta(f)<c n^{-\left(v_{H}-2\right) /\left(e_{H}-1\right)}\right]=\Omega\left(\frac{(\ln c)^{1 /\left(e_{H}-1\right)}}{c}\right),
$$

which, given the discussion above, proves Lemma 2.2. In order to prove (17) it would be convenient to first restrict ourselves to the following special case. Consider the tree $R T_{f, d}$ which is obtained from $T_{f, d}$ as follows: For every non-leaf node $g$ at even height in $T_{f, d}$, remove an arbitrary subset of the subtrees rooted at the nodes adjacent to $g$, so that the outdegree of $g$ becomes $\left\lfloor\lambda k^{e_{H}-1}\right\rfloor-\left\lfloor k^{e_{H} / 2-1 / 3}\right\rfloor$ exactly. Note that this can be done, as we assume that $E_{1}$ occurs. For a node $g$ at height $2 d$ in $R T:=R T_{f, D}$, we denote by $R T_{g, d}$ the subtree of $R T$ that is rooted at $g$. Given this definition the task of lower bounding (16) is now divided to two parts. In the first part (Section 4.1), we prove (17) for the special case that $T_{f, D}$ is replaced with $R T_{f, D}$. In the second part (Section 4.2), we show that asymptotically, the probability of $\left\{\overline{B\left(T_{f, D}\right)} \mid \beta(f)<c n^{-\left(v_{H}-2\right) /\left(e_{H}-1\right)}\right\}$ is equal

to the probability of $\left\{\overline{B\left(R T_{f, D}\right)} \mid \beta(f)<c n^{-\left(v_{H}-2\right) /\left(e_{H}-1\right)}\right\}$. Combining these two parts, we will conclude the validity of (17) and so also the validity of Lemma 2.2.

\subsection{Analyzing $\overline{B(R T)}$}

For $x<y$, let $p_{d}(x, y)$ be the probability of $\overline{B\left(R T_{f, d}\right)}$ conditioned on $\left\{x n^{-\left(v_{H}-2\right) /\left(e_{H}-1\right)} \leq\right.$ $\left.\beta(f)<y n^{-\left(v_{H}-2\right) /\left(e_{H}-1\right)}\right\}$. The main result in this subsection follows.

Lemma 4.2. $p_{D}(0, c)=\Omega\left(\frac{(\ln c)^{1 /\left(e_{H}-1\right)}}{c}\right)$.

To prove Lemma 4.2, we begin with the following proposition.

Proposition 4.3. For $0<x \leq c$ and $n$ sufficiently large, $p_{D}(0, x) \geq p_{D-1}(0, x)-2^{-D+1}$.

Proof. Define

$\mathcal{G}_{i}:= \begin{cases}\left\{G_{1} \in \Gamma_{R T}(f): \forall g_{1} \in G_{1} . \beta\left(g_{1}\right)<c n^{-\left(v_{H}-2\right) /\left(e_{H}-1\right)}\right\} & \text { if } i=1, \\ \bigcup_{g_{i-1} \in G_{i-1} \in \mathcal{G}_{i-1}}\left\{G_{i} \in \Gamma_{R T}\left(g_{i-1}\right): \forall g_{i} \in G_{i} . \beta\left(g_{i}\right)<\beta\left(g_{i-1}\right)\right\} & \text { if } 2 \leq i \leq d .\end{cases}$ 
By inspecting Definition 5, one sees that conditioned on $\left\{\beta(f)<x n^{-\left(v_{H}-2\right) /\left(e_{H}-1\right)}\right\}$, $\overline{B\left(R T_{f, D-1}\right)}$ implies $\overline{B\left(R T_{f, D}\right)} \cup\left\{\mathcal{G}_{D-1} \neq \emptyset\right\}$. Hence $p_{D-1}(0, x) \leq p_{D}(0, x)+\operatorname{Pr}\left[\mathcal{G}_{D-1} \neq \emptyset\right]$ and so it suffices to prove that $\operatorname{Pr}\left[\mathcal{G}_{D-1} \neq \emptyset\right] \leq 2^{-D+1}$. For that, we show that the expected size of $\mathcal{G}_{D-1}$ is at most $2^{-D+1}$.

To estimate the expected size of $\mathcal{G}_{D-1}$, we first give an upper bound on the probability that a given subgraph $G_{D-1}$ is included in the set $\mathcal{G}_{D-1}$. For that, let us consider the unique path $\left(f=g_{0}, G_{1}, g_{1}, \ldots, g_{D-2}, G_{D-1}\right)$ from the root of $R T_{f, D}$ to $G_{D-1}$. Then $G_{1}$ is included in $\mathcal{G}_{1}$ if and only if $\beta(g)<c n^{-\left(v_{H}-2\right) /\left(e_{H}-1\right)}$ for every $g \in G_{1}$. For $j \geq 2$, $G_{j}$ is included in $\mathcal{G}_{j}$ if and only if $G_{i}$ is included in $\mathcal{G}_{i}$ for every $i<j$ and in addition, $\beta(g)<\beta\left(g_{j-1}\right)$ for every $g \in G_{j}$. Now, the probability of $\left\{G_{1} \in \mathcal{G}_{1}\right\}$ is exactly $(c / k)^{e_{H}-1}$. Given $G_{1} \in \mathcal{G}_{1}$, we have that $g_{1}$ is uniformly distributed in $\left[0, c n^{-\left(v_{H}-2\right) /\left(e_{H}-1\right)}\right)$. Moreover, for $j \geq 2$, given that $G_{j} \in \mathcal{G}_{j}$, we have that $g_{j}$ is uniformly distributed in $\left[0, \beta\left(g_{j-1}\right)\right.$ ). Hence, it follows that

$$
\begin{aligned}
\operatorname{Pr}\left[G_{D-1} \in \mathcal{G}_{D-1}\right] & =\int_{0}^{1} \ldots \int_{0}^{1}\left(\frac{c}{k}\right)^{e_{H}-1} \cdot \prod_{j=2}^{D-1}\left(\frac{c}{k} \cdot \prod_{i=1}^{j-1} x_{i}\right)^{e_{H}-1} d x_{1} d x_{2} \ldots d x_{D-2} \\
& =\frac{c^{\left(e_{H}-1\right)(D-1)}}{k^{\left(e_{H}-1\right)(D-1)}} \cdot \frac{1}{\prod_{i=1}^{D-2}\left(i\left(e_{H}-1\right)+1\right)} \\
& <\frac{c^{\left(e_{H}-1\right)(D-1)}}{k^{\left(e_{H}-1\right)(D-1)}(D-1) !}
\end{aligned}
$$

where the inequality follows from $e_{H} \geq 3$. Since there are no more than $\left(e_{H} \lambda k^{e_{H}-1}\right)^{D-1}$ nodes at distance $2(D-1)-1$ from the root of $R T$, we deduce from (18) that the expected number of nodes in $\mathcal{G}_{D-1}$ is at most $\left(e_{H} \lambda c^{e_{H}-1}\right)^{D-1} /((D-1) !)$. Since $(D-1) ! \geq$ $((D-1) / 3)^{D-1}$ and since $D-1 \geq 6 e_{H} \lambda c^{e_{H}-1}$ for $n$ sufficiently large, the expected number of nodes in $\mathcal{G}_{D-1}$ is at most

$$
\frac{\left(3 e_{H} \lambda c^{e_{H}-1}\right)^{D-1}}{(D-1)^{D-1}} \leq \frac{\left(3 e_{H} \lambda c^{e_{H}-1}\right)^{D-1}}{\left(6 e_{H} \lambda c^{e_{H}-1}\right)^{D-1}}=2^{-D+1},
$$

as required. This completes the proof.

We also need the following fact.

Proposition 4.4. Let $x<y$. Then $p_{d}(0, x) \geq p_{d}(0, y)$ and $p_{d}(0, x) \geq p_{d}(x, y)$.

Proof. Since $p_{d}(0, y)$ is the weighted average of $p_{d}(0, x)$ and $p_{d}(x, y)$, it is enough to show that $p_{d}(0, x) \geq p_{d}(x, y)$. Take any birthtime function $\beta$ under which $\overline{B\left(R T_{f, d}\right)}$ occurs, with $x n^{-\left(v_{H}-2\right) /\left(e_{H}-1\right)} \leq \beta(f)<y n^{-\left(v_{H}-2\right) /\left(e_{H}-1\right)}$. Now alter $\beta(f)$ so that we have $\beta(f)<$ $x n^{-\left(v_{H}-2\right) /\left(e_{H}-1\right)}$. It is easy to check given Definition 5 that $\overline{B\left(R T_{f, d}\right)}$ occurs even after the above alteration of $\beta$. This implies that $p_{d}(0, x) \geq p_{d}(x, y)$.

We now turn to lower bound $p_{D}(0, c)$. Let $g \in G \in \Gamma_{R T}(f)$. Condition on the occurrence of $\left\{i n^{-\left(v_{H}-2\right) /\left(e_{H}-1\right)} \leq \beta(f)<(i+1) n^{-\left(v_{H}-2\right) /\left(e_{H}-1\right)}\right\}$ for some $i \in[c-1]$. 
Under that condition we have $\operatorname{Pr}[\beta(g)<\beta(f)] \leq(i+1) / k$. If we further condition on $\{\beta(g)<\beta(f)\}$ then we have by Proposition 4.4 (and the fact that $R T_{g, D-1}$ is isomorphic to $\left.T_{f, D-1}\right)$ that the probability of $\overline{B\left(R T_{g, D-1}\right)}$ is upper bounded by $p_{D-1}(0, i)$. From these observations, using Definition 5 and the fact that $R T_{f, D}$ is a subtree of the good tree $T_{f, D}$, we get that for all $i \in[c-1]$,

$$
p_{D}(i, i+1) \geq\left(1-\left(\frac{(i+1) \cdot p_{D-1}(0, i)}{k}\right)^{e_{H}-1}\right)^{\left\lfloor\lambda k^{e_{H}-1}\right\rfloor-\left\lfloor k^{e_{H} / 2-1 / 3}\right\rfloor} .
$$

Hence, by Proposition 4.3 we get

$$
\begin{aligned}
p_{D}(0, c) & \geq \frac{1}{c} \sum_{i=\lceil c / 2\rceil}^{c-1} p_{D}(i, i+1) \\
& \geq \frac{1}{c} \sum_{i=\lceil c / 2\rceil}^{c-1}\left(1-\left(\frac{(i+1) \cdot p_{D-1}(0, i)}{k}\right)^{e_{H}-1}\right)^{\left\lfloor\lambda k^{e_{H}-1}\right\rfloor-\left\lfloor k^{e_{H} / 2-1 / 3}\right\rfloor} \\
& \geq \frac{1}{c} \sum_{i=\lceil c / 2\rceil}^{c-1}\left(1-\left(\frac{(i+1) \cdot\left(p_{D}(0, i)+2^{-D+1}\right)}{k}\right)^{e_{H}-1}\right)^{\left\lfloor\lambda k^{e_{H}-1}\right\rfloor-\left\lfloor k^{e_{H} / 2-1 / 3}\right\rfloor} .
\end{aligned}
$$

Define

$$
\tau(i)=\frac{\left((100 \lambda)^{-1} \ln i\right)^{1 /\left(e_{H}-1\right)}}{i+1}-2^{-D+1} .
$$

We have two cases. First assume that $p_{D}(0, i) \geq \tau(i)$ for some integer $i,\lceil c / 2\rceil \leq i \leq c-1$. In that case the proof is complete, since by Proposition 4.4, we get

$$
p_{D}(0, c) \geq \frac{p_{D}(0,\lceil c / 2\rceil)}{2} \geq \frac{p_{D}(0, i)}{2} \geq \frac{\tau(i)}{2}=\Omega\left(\frac{(\ln c)^{1 /\left(e_{H}-1\right)}}{c}\right) .
$$

Next, assume that $p_{D}(0, i)<\tau(i)$ for all integers $i,\lceil c / 2\rceil \leq i \leq c-1$. In that case, by replacing $p_{D}(0, i)$ with $\tau(i)$ in the sum above, we get

$$
p_{D}(0, c) \geq \frac{1}{c} \sum_{i=\lceil c / 2\rceil}^{c-1}\left(1-\frac{\ln i}{100 \lambda k^{e_{H}-1}}\right)^{\lambda k^{e_{H}-1}}=\Omega\left(\frac{(\ln c)^{1 /\left(e_{H}-1\right)}}{c}\right) .
$$

Note that Lemma 4.2 gives the validity of (17) for the case where $T_{f, D}$ is replaced with $R T_{f, D}$. In the next subsection we show that asymptotically, the probability of $\overline{B\left(T_{f, D}\right)}$ conditioned on $\left\{\beta(f)<c n^{-\left(v_{H}-2\right) /\left(e_{H}-1\right)}\right\}$ is equal to $p_{D}(0, c)$. This will prove (17) and hence also Lemma 2.2.

\subsection{On $\overline{B(R T)}$ versus $\overline{B(T)}$}

For $x<y$ and for a node $g$ at height $2 d$ in $T$, let $q_{g, d}(x, y)$ be the probability of $\overline{B\left(T_{g, d}\right)}$ conditioned on $\left\{x n^{-\left(v_{H}-2\right) /\left(e_{H}-1\right)} \leq \beta(g)<y n^{-\left(v_{H}-2\right) /\left(e_{H}-1\right)}\right\}$. The main result in this subsection follows. 
Lemma 4.5. $(1-o(1)) \cdot p_{D}(0, c) \leq q_{f, D}(0, c) \leq(1+o(1)) \cdot p_{D}(0, c)$.

Note that Lemma 4.5 together with Lemma 4.2 implies (17), which in turn implies Lemma 2.2. We begin the proof of Lemma 4.5 by giving a few useful definitions which we use throughout the proof.

\section{Definition 6 .}

- Define for every integer $d \leq D$,

$$
\varepsilon_{d}:=\frac{\left(128 e_{H} c\right)^{2 d e_{H}}}{k^{e_{H} / 2-2 / 3}}
$$

- Fix $\delta>0$ which satisfies the following: (i) $\delta \leq k^{-20 e_{H}}$, (ii) $\delta^{1 / 4}=o\left(\varepsilon_{d} \cdot p_{d}(0, c)\right.$ ) for all $d \in[D]$, (iii) $\left(1-\left(2 \delta^{1 / 4} / k\right)^{e_{H}-1}\right)^{2 k^{e H^{-1}}} \geq 1-\varepsilon_{d} / 2$ for all $d \in[D]$, and (iv) $1 / \sqrt{\delta} \in \mathbb{N}$.

- Define $J:=\{\sqrt{\delta}, \sqrt{\delta}+\delta, \sqrt{\delta}+2 \delta, \ldots, c-2 \delta, c-\delta\}$.

Proposition 4.6. Let $g$ be a node at height $2 d$ in $T, 0 \leq d \leq D$. Assume that for all $j \in J$,

$$
\left(1-\varepsilon_{d} / 2\right) \cdot p_{d}(j, j+\delta) \leq q_{g, d}(j, j+\delta) \leq\left(1+\varepsilon_{d} / 2\right) \cdot p_{d}(j, j+\delta)
$$

Then for all $j \in J \cup\{c\}$,

$$
\left(1-\varepsilon_{d}\right) \cdot p_{d}(0, j) \leq q_{g, d}(0, j) \leq\left(1+\varepsilon_{d}\right) \cdot p_{d}(0, j) .
$$

Proof. First note that the conclusion in the proposition is trivially true for $d=0$, since $q_{g, d}(0, j)=p_{d}(0, j)=1$ for all $j \in J \cup\{c\}$. Hence, we may assume that $d \geq 1$.

Fix $j \in J \cup\{c\}$. Assume first that $j>\sqrt{\delta}+\delta^{1 / 4}$. Trivially we have:

$$
\begin{aligned}
p_{d}(0, j) & =O\left(\frac{\sqrt{\delta}}{j}\right)+\frac{\delta}{j} \cdot \sum_{j^{\prime} \in J: j^{\prime}<j} p_{d}\left(j^{\prime}, j^{\prime}+\delta\right), \\
q_{g, d}(0, j) & =O\left(\frac{\sqrt{\delta}}{j}\right)+\frac{\delta}{j} \cdot \sum_{j^{\prime} \in J: j^{\prime}<j} q_{g, d}\left(j^{\prime}, j^{\prime}+\delta\right) .
\end{aligned}
$$

Since $j>\sqrt{\delta}+\delta^{1 / 4}$ we have $\sqrt{\delta} / j \leq \delta^{1 / 4}$. By definition, $\delta^{1 / 4}=o\left(\varepsilon_{d} \cdot p_{d}(0, c)\right)$. By Proposition 4.4 and since $j \leq c$, we have $p_{d}(0, c) \leq p_{d}(0, j)$. Therefore, $\sqrt{\delta} / j \leq \delta^{1 / 4}=$ $o\left(\varepsilon_{d} \cdot p_{d}(0, j)\right)$. This, it can be verified, together with the two equalities above and with the assumptions given in the proposition, implies the validity of the claim for $j$.

Next, assume $j \leq \sqrt{\delta}+\delta^{1 / 4}$. Crudely, $q_{g, d}(0, j)$ is lower bounded by the probability of the event that for every $G \in \Gamma_{T}(g)$ there is an edge $g^{\prime} \in G$ with $\left\{\beta\left(g^{\prime}\right)>\beta(g)\right\}$. Hence, since $\left|\Gamma_{T}(g)\right| \leq 2 k^{e_{H}-1}$ and by definition of $\delta$,

$$
q_{g, d}(0, j) \geq\left(1-\left(\frac{2 \delta^{1 / 4}}{k}\right)^{e_{H}-1}\right)^{2 k^{e} H^{-1}} \geq 1-\varepsilon_{d} / 2 .
$$


Trivially, $p_{d}(0, j) \leq 1$. Hence, it follows that $q_{g, d}(0, j)$ is lower bounded by $\left(1-\varepsilon_{d}\right) \cdot p_{d}(0, j)$, as required. The argument for the upper bound is similar. Trivially, $q_{g, d}(0, j) \leq 1$. Also, by an argument similar to the one used above, $p_{d}(0, j) \geq 1-\varepsilon_{d} / 2$. Hence, one can verify that indeed $q_{g, d}(0, j) \leq\left(1+\varepsilon_{d}\right) \cdot p_{d}(0, j)$. Thus, the claim is valid for all $j \in J \cup\{c\}$.

The following proposition, when combined with Proposition 4.6, implies Lemma 4.5.

Proposition 4.7. Let $g$ be a node at height $2 d$ in $T, 0 \leq d \leq D$. Then for all $j \in J$,

$$
\left(1-\varepsilon_{d} / 2\right) \cdot p_{d}(j, j+\delta) \leq q_{g, d}(j, j+\delta) \leq\left(1+\varepsilon_{d} / 2\right) \cdot p_{d}(j, j+\delta)
$$

The rest of the paper is dedicated for the proof of Proposition 4.7. We collect some useful facts:

Claim 4.8. Let $g$ be a node at height $2 d$ in $T, 1 \leq d \leq D$. Then for all $j \in J$,

(1) $q_{g, d}(j, j+\delta) \leq \prod_{G \in \Gamma_{T}(g)}\left(1-\prod_{g^{\prime} \in G} \frac{j \cdot q_{g^{\prime}, d-1}(0, j)}{k}\right)$.

(2) $q_{g, d}(j, j+\delta) \geq\left(1-\varepsilon_{d-1}\right) \cdot \prod_{G \in \Gamma_{T}(g)}\left(1-\prod_{g^{\prime} \in G} \frac{j \cdot q_{g^{\prime}, d-1}(0, j)}{k}\right)$.

(3) $p_{d}(j, j+\delta) \geq\left(1+\varepsilon_{d-1}\right)^{-1} \cdot\left(1-\left(\frac{j \cdot p_{d-1}(0, j)}{k}\right)^{e_{H}-1}\right)^{\left|\Gamma_{R T}(f)\right|}$.

(4) $p_{d}(j, j+\delta) \leq\left(1-\left(\frac{j \cdot p_{d-1}(0, j)}{k}\right)^{e_{H}-1}\right)^{\left|\Gamma_{R T}(f)\right|}$.

Proof. (1) Condition on $\left\{j n^{-\left(v_{H}-2\right) /\left(e_{H}-1\right)} \leq \beta(g)<(j+\delta) n^{-\left(v_{H}-2\right) /\left(e_{H}-1\right)}\right\}$ and fix $g^{\prime} \in G \in \Gamma_{T}(g)$. Given the definition of $\overline{B\left(T_{g, d}\right)}$ and the fact that $T$ is a good tree, it is enough to verify that the probability of the event $\left\{\beta\left(g^{\prime}\right)<\beta(g)\right\} \cap \overline{B\left(T_{g^{\prime}, d-1}\right)}$ is at least $j \cdot q_{g^{\prime}, d-1}(0, j) / k$. Indeed, the event in question is implied by the occurrence of $\left\{\beta\left(g^{\prime}\right)<j n^{-\left(v_{H}-2\right) /\left(e_{H}-1\right)}\right\} \cap \overline{B\left(T_{g^{\prime}, d-1}\right)}$.

(2) Let $\mathcal{E}_{1}$ be the event that for all $G \in \Gamma_{T}(g)$, it does not hold that for all $g^{\prime} \in$ $G$, both $\left\{\beta\left(g^{\prime}\right)<j n^{-\left(v_{H}-2\right) /\left(e_{H}-1\right)}\right\}$ and $\overline{B\left(R T_{g^{\prime}, d-1}\right)}$ occur. Let $\mathcal{E}_{2}$ be the event that for all $g^{\prime} \in G \in \Gamma_{T}(g), \beta\left(g^{\prime}\right)$ is not in the interval $\left[j n^{-\left(v_{H}-2\right) /\left(e_{H}-1\right)},(j+\right.$ $\left.\delta) n^{-\left(v_{H}-2\right) /\left(e_{H}-1\right)}\right)$. It is easy to verify that $q_{g, d}(j, j+\delta) \geq \operatorname{Pr}\left[\mathcal{E}_{1} \cap \mathcal{E}_{2}\right]$. Now, we have $\operatorname{Pr}\left[\mathcal{E}_{1}\right]=\prod_{G \in \Gamma_{T}(g)}\left(1-\prod_{g^{\prime} \in G} \frac{j \cdot q_{g^{\prime}, d-1}(0, j)}{k}\right)$. Given $\mathcal{E}_{1}$, every edge $g^{\prime} \in G \in \Gamma_{T}(g)$ either satisfies $\beta\left(g^{\prime}\right)<j n^{-\left(v_{H}-2\right) /\left(e_{H}-1\right)}$ or else, $\beta\left(g^{\prime}\right)$ is uniformly distributed in the interval $\left[j n^{-\left(v_{H}-2\right) /\left(e_{H}-1\right)}, k n^{-\left(v_{H}-2\right) /\left(e_{H}-1\right)}\right]$. Hence, since the number of choices for $g^{\prime} \in G \in \Gamma_{T}(g)$ is at most $2 \lambda e_{H} k^{e_{H}-1}$, for $n$ sufficiently large, $\operatorname{Pr}\left[\mathcal{E}_{2} \mid \mathcal{E}_{1}\right] \geq$ $(1-2 \delta / k)^{2 \lambda e_{H} k^{e_{H}-1}}$ which is at least $1-\varepsilon_{d-1}$ by the choice of $\delta$. This proves the claim.

(3) The proof is similar to the proof of Claim 4.8 (2).

(4) The proof is similar to the proof of Claim 4.8 (1). 
We continue with the proof of Proposition 4.7, which is by induction on $d$. The base case, $d=0$ is trivially true, since $q_{g, 0}(j, j+\delta)=p_{0}(j, j+\delta)=1$ for all $j \in J$. Fix $d \in[D]$, assume that the proposition holds for $d-1$ and let $j \in J$. In the argument that follows, we use implicitly the fact that for $y>1$, $\exp (-1 /(y-1))<1-1 / y<\exp (-1 / y)$.

By Claim 4.8 (1), the induction hypothesis and Proposition 4.6 and by the occurrence of $E_{1}$ we have

$$
\begin{aligned}
q_{g, d}(j, j+\delta) & \leq \prod_{G \in \Gamma_{T}(g)}\left(1-\prod_{g^{\prime} \in G} \frac{j \cdot q_{g^{\prime}, d-1}(0, j)}{k}\right) \\
& \leq \prod_{G \in \Gamma_{T}(g)}\left(1-\prod_{g^{\prime} \in G} \frac{\left(1-\varepsilon_{d-1}\right) \cdot j \cdot p_{d-1}(0, j)}{k}\right) \\
& \leq\left(1-\left(\frac{\left(1-\varepsilon_{d-1}\right) \cdot j \cdot p_{d-1}(0, j)}{k}\right)^{e_{H}-1}\right)^{\left\lfloor\lambda k^{e_{H}-1}\right\rfloor-\left\lfloor k^{e_{H} / 2-1 / 3}\right\rfloor}=(*) .
\end{aligned}
$$

Using Claim 4.8 (3) we can further upper bound $(*)$ as follows:

$$
\begin{aligned}
(*) & \leq\left(1-\left(\frac{j \cdot p_{d-1}(0, j)}{k}\right)^{e_{H}-1}\right)^{\left(\left\lfloor\lambda k^{e_{H}-1}\right\rfloor-\left\lfloor k^{e_{H} / 2-1 / 3}\right\rfloor\right)\left(1-2 e_{H} \varepsilon_{d-1}\right)} \\
& \leq\left(1-\left(\frac{j \cdot p_{d-1}(0, j)}{k}\right)^{e_{H}-1}\right)^{\left\lfloor\lambda k^{e_{H}-1}\right\rfloor-\left\lfloor k^{e_{H} / 2-1 / 3}\right\rfloor-2 e_{H} \varepsilon_{d-1} \lambda k^{e_{H}-1}} \\
& \leq\left(1+\varepsilon_{d-1}\right) \cdot p_{d}(j, j+\delta) \cdot\left(1-\left(\frac{j}{k}\right)^{e_{H}-1}\right)^{-2 e_{H} \varepsilon_{d-1} \lambda k^{e_{H}-1}} \\
& \leq\left(1+\varepsilon_{d} / 2\right) \cdot p_{d}(j, j+\delta) .
\end{aligned}
$$

Now, by Claim 4.8 (2), the induction hypothesis and Proposition 4.6 and by the occurrence of $E_{1}$ we have

$$
\begin{aligned}
q_{g, d}(j, j+\delta) & \geq\left(1-\varepsilon_{d-1}\right) \cdot \prod_{G \in \Gamma_{T}(g)}\left(1-\prod_{g^{\prime} \in G} \frac{j \cdot q_{g^{\prime}, d-1}(0, j)}{k}\right) \\
& \geq\left(1-\varepsilon_{d-1}\right) \cdot \prod_{G \in \Gamma_{T}(g)}\left(1-\prod_{g^{\prime} \in G} \frac{\left(1+\varepsilon_{d-1}\right) \cdot j \cdot p_{d-1}(0, j)}{k}\right) \\
& \geq\left(1-\varepsilon_{d-1}\right) \cdot\left(1-\left(\frac{\left(1+\varepsilon_{d-1}\right) \cdot j \cdot p_{d-1}(0, j)}{k}\right)^{e_{H}-1}\right)^{\lambda k^{e} H^{-1}+k^{e} H^{2-1 / 3}}=(* *) .
\end{aligned}
$$

One can now use Claim 4.8 (4) to further lower bound $(* *)$ as follows:

$$
\begin{aligned}
(* *) & \geq\left(1-\varepsilon_{d-1}\right) \cdot\left(1-\left(\frac{j \cdot p_{d-1}(0, j)}{k}\right)^{e_{H}-1}\right)^{\left(\lambda k^{e_{H}-1}+k^{e_{H} / 2-1 / 3}\right)\left(1+2 e_{H} \varepsilon_{d-1}\right)} \\
& \geq\left(1-\varepsilon_{d-1}\right) \cdot\left(1-\left(\frac{j \cdot p_{d-1}(0, j)}{k}\right)^{e_{H}-1}\right)^{\left\lfloor\lambda k^{e_{H}-1}\right\rfloor-\left\lfloor k^{e_{H} / 2-1 / 3}\right\rfloor+8 e_{H} \varepsilon_{d-1} \lambda k^{e_{H}-1}} \\
& \geq\left(1-\varepsilon_{d-1}\right) \cdot p_{d}(j, j+\delta) \cdot\left(1-\left(\frac{j}{k}\right)^{e_{H}-1}\right)^{8 e_{H} \varepsilon_{d-1} \lambda k^{e_{H}-1}} \\
& \geq\left(1-\varepsilon_{d} / 2\right) \cdot p_{d}(j, j+\delta) .
\end{aligned}
$$

This completes the proof of Proposition 4.7. 


\section{Acknowledgment}

The author would like to kindly thank Ilan Newman and Oren Ben-Zwi for helpful discussions.

\section{References}

[1] T. Bohman, The triangle-free process (2008), arXiv:0806.4375v1.

[2] B. Bollobás and O. Riordan, Constrained graph processes, Electr. J. Comb. 7 (2000).

[3] W. G. Brown, On graphs that do not contain a Thomsen graph, Canad. Math. Bull. 9 (1966), 281-285.

[4] P. Erdős, A. Rényi, and V. T. Sós, On a problem of graph theory, Studia Sci. Acad. Math. Hungar. 1 (1966), 215-235.

[5] P. Erdős and J. H. Spencer, Probabilistic methods in combinatorics, 1974.

[6] P. Erdős, S. Suen, and P. Winkler, On the size of a random maximal graph, Random Struct. Algorithms 6 (1995), 309-318.

[7] S. Janson, T. Luczak, and A. Ruciński, Random graphs, 2000.

[8] S. Janson, Poisson approximation for large deviations, Random Struct. Algorithms 1 (1990), 221-230.

[9] D. Osthus and A. Taraz, Random maximal h-free graphs, Random Struct. Algorithms 18 (2001), 61-82.

[10] A. Rucinski and N. C. Wormald, Random graph processes with degree restrictions, Combinatorics, Probability \& Computing 1 (1992), 169-180.

[11] J. H. Spencer, Threshold functions for extension statements, J. Comb. Theory, Ser. A 53 (1990), 286-305.

[12] _ Maximal triangle-free graphs and Ramsey $r(3, t)$ (unpublished manuscript, 1995). 